\title{
A multi-scale model for solute transport in a wavy-walled channel
}

\author{
H. F. Woollard · J. Billingham • O. E. Jensen • \\ G. Lian
}

Received: 26 February 2008 / Accepted: 21 July 2008 / Published online: 3 September 2008

(C) The Author(s) 2008. This article is published with open access at Springerlink.com

\begin{abstract}
This paper concerns steady flow and solute uptake in a wavy-walled channel, where the wavelength and amplitude of the wall are comparable to each other but are much shorter than the width of the channel. The problem has two primary asymptotic regions: a core region where the walls appear flat at leading order and a wall region where there is full interaction between advection, diffusion and uptake at the wavy wall. For weak wall uptake, the effective uptake from the core is shown to increase with wall waviness in proportion to surface area, whereas for stronger wall uptake, it is found that the uptake from the core can be reduced as the wall amplitude increases. Conditions are identified under which this approximation is uniformly valid in a full channel flow, accounting for inlet conditions, and a comprehensive survey of the asymptotic distributions of solute both along and across the channel is provided. It is also shown how this multiscale approach can readily be extended to account for channel walls with multiple lengthscales of spatial variation.
\end{abstract}

Keywords Advection-diffusion $\cdot$ Matched asymptotic expansions $\cdot$ Solute uptake $\cdot$ Viscous flow

\section{Introduction}

In this paper we study flow and solute uptake in a channel with wavy walls, where the amplitude and wavelength of the walls are comparable to each other but small compared to the channel width. This situation occurs in biological systems, for example the small intestine (where the wall is covered with small projections called villi) and blood vessels (where nuclei of endothelial cells intrude into the lumen). Similar flows can also be found in widespread physical systems with surface roughness. By examining flow and transport at the micro-scale (that of the surface roughness), we derive effective boundary conditions for flow and transport at the macro-scale (that of the channel).

There are a large number of prior studies on flow over rough or wavy surfaces, both analytical and experimental. Many have focused on the role of wall roughness in the context of the no-slip boundary condition, an issue of increasing interest in microfluidic applications [1]; the roughness induces in its far field a shift in the velocity profile by a distance equivalent to an effective slip length, which depends on the size and shape of the roughness. For a

H. F. Woollard · J. Billingham · O. E. Jensen ( $\varangle)$

School of Mathematical Sciences, University of Nottingham, University Park, Nottingham NG7 2RD, UK

e-mail: Oliver.Jensen@nottingham.ac.uk

G. Lian

Unilever R\&D Colworth, Colworth House, Sharnbrook, Bedford MK44 1LQ, UK 
review of experimental work in this area see [2]. There have been numerous theoretical approaches to this problem using a variety of methods, with early studies motivated by the problem of defining an effective boundary condition at the edge of a porous medium [3-5]. In particular, Hocking [6] computed the 2D viscous shear flow over a wavy surface, finding an amplitude-dependent slip for small-amplitude roughness (using a series solution method) and a limiting slip for effectively infinite-amplitude roughness (using a Weiner-Hopf technique). Hocking's work has subsequently been extended to account for inertial effects [7,8], non-sinusoidal roughness [9-11], surface coatings [12], randomly distributed protuberances [13] or random surface corrugations [14] and grooves oriented relative to the flow [15,16]. At zero Reynolds number, the streamlines in sufficiently deep grooves exhibit flow reversal [17], a feature which is enhanced by inertia $[18,19]$. Stokes flows over wavy surfaces have been addressed with a variety of theoretical approaches, including boundary-integral methods [20,21], conformal-mapping techniques [22] and asymptotic homogenisation approaches $[23,24]$.

Transport over rough surfaces by diffusion alone has been investigated in the context of fractal surfaces, where uptake at the wall has been shown to be dominated by the large-scale shape of the wall, being less sensitive to the small-scale structures [25,26]. Likewise, Fyrillas and Pozrikidis [27] showed that diffusive transport across an irregular surface does not scale with surface area, but asymptotes as the amplitude of the irregularities increases. Below we revisit these multi-scale issues, accounting for the presence of flow. The optimisation of surfaces for engineering heat-transfer applications [28] raises interesting analogous questions in biological contexts such as the gut (where villi are covered with yet finer corrugations called microvilli), where numerous additional constraints must be observed. Transport over irregular surfaces by flow alone can exhibit mixing by chaotic advection [29,30], when the Hamiltonian structure of 2D Stokes flow is perturbed. Transport by Stokes drift over wavy surfaces has also been described [31].

There is in addition a substantial literature on high-Reynolds-number heat and mass transfer in corrugated channels and pipes [32-34], which we do not attempt to summarise here. However, certain features are relevant to our study, for example increased uptake at the peaks of corrugations [35] and saturation and possible decrease of uptake with increased waviness [36].

The present study seeks to fill an important gap in the existing literature, describing transport by Stokes flow in a wavy-walled channel in the presence of diffusion, allowing for uptake of solute at the wall. While effective slip conditions for the flow are well characterised, equivalent conditions for uptake of a solute are not. Rather than follow a formal homogenisation approach [37], we exploit matched asymptotic expansions in the limit in which the wall wavelength and amplitude are both small compared to the channel width. The model is outlined in Sect. 2; flow results are reviewed in Sect. 3 and the derivation of the effective uptake parameter is described in Sect. 4. In treating the inner problem at the wall computationally, instead of mapping the wavy domain to a plane surface [38], we compute the flow and transport directly with a finite-element method. In addition to a primary inner layer along the wavy wall matching an outer layer in the core of the channel (for formal convergence of related problems see [39]), we identify a range of other asymptotic regions within and along the channel whose dimensions are functions of the effective uptake parameter and Péclet number (Sect. 5). By deriving an effective uptake parameter, and identifying the global conditions under which it is appropriate, we show how (and when) to approximate flows in walls with multiple lengthscales of waviness. We illustrate the utility of the approach in Sect. 6 by considering flow in a channel which has two levels of waviness at its wall.

\section{Mathematical model}

We consider a long, planar, wavy-walled channel of mean width $2\left(a^{*}+\varepsilon \lambda^{*}\right)$, symmetric about its midline $Y^{*}=a^{*}$ (* denotes a dimensional quantity). The channel walls have wavelength $2 \pi \lambda^{*}$ and amplitude $\varepsilon \lambda^{*}$, where $\lambda^{*} \ll a^{*}$, $\varepsilon=O(1)$ and the lower wall lies at

$Y^{*}=\varepsilon \lambda^{*}\left(\cos \left(X^{*} / \lambda^{*}\right)-1\right) \equiv a^{*} F\left(X^{*} / a^{*} ; \lambda^{*} / a^{*}, \varepsilon\right)$, 
where $X^{*}$ measures distance down the channel. A viscous fluid flows through the channel transporting a solute which is absorbed through the walls via a mixed boundary condition. At the inlet the flow has a Poiseuille profile and provides a steady influx of solute. The steady Stokes flow down the channel satisfies

$\nabla^{*} P^{*}=\eta^{*} \nabla^{* 2} \mathbf{U}^{*}, \quad \nabla^{*} \cdot \mathbf{U}^{*}=0, \quad\left(a^{*} F \leq Y^{*} \leq a^{*}, \quad X^{*}>0\right)$,

subject to boundary conditions

$\mathbf{U}^{*}=\mathbf{0}$ on $Y^{*}=a^{*} F, \quad \boldsymbol{U}_{Y^{*}}^{*}=\mathbf{0}, \boldsymbol{V}^{*}=\mathbf{0}$ on $Y^{*}=a^{*}$,

$\mathbf{U}^{*}=U_{0}^{*}\left(Y^{*}\left(2 a^{*}-Y^{*}\right) / a^{* 2}, 0\right)$ on $X^{*}=0$.

Here $U_{0}^{*}$ is the maximum axial velocity down the channel and $\eta^{*}$ is the viscosity of the fluid. Solute transport is governed by the steady advection-diffusion equation

$\left(\mathbf{U}^{*} \cdot \nabla^{*}\right) C^{*}=D^{*} \nabla^{2} C^{*} \quad$ for $\quad a^{*} F \leq Y^{*} \leq a^{*}, \quad X^{*}>0$,

subject to boundary conditions

$\left(\mathbf{n} \cdot \nabla^{*}\right) C^{*}=-\mu^{*} C^{*}$ on $Y^{*}=a^{*} F, \quad C_{Y^{*}}^{*}=0$ on $Y^{*}=a^{*}$,

$C^{*}=C_{0}^{*}$ on $X^{*}=0$,

where $D^{*}$ is the solute diffusivity, $C_{0}^{*}$ is the uniform solute concentration at the inlet of the channel, $\mathbf{n}$ is the unit outward normal on $Y^{*}=a^{*} F$ and $\mu^{*}>0$ is an uptake parameter (so that $D^{*} \mu^{*}$ is the mass-transfer coefficient across the wall). We assume that the channel is long enough for the concentration to tend to zero far downstream.

We non-dimensionalise the problem using

$\mathbf{U}^{*}=2 U_{0}^{*} \mathbf{U}, \quad C^{*}=C_{0}^{*} C$,

$P^{*}=\left(2 \eta^{*} U_{0}^{*} / a^{*}\right) P, \quad Y^{*}=a^{*} Y, \quad X^{*}=a^{*} X$,

which gives a unit dimensionless shear rate near the wall. Equations (2.2-2.5) become

$\nabla P=\nabla^{2} \mathbf{U}, \quad \nabla \cdot \mathbf{U}=0$ for $F \equiv \varepsilon \lambda(\cos (X / \lambda)-1) \leq Y \leq 1, X>0$,

$\mathbf{U}=\mathbf{0}$ on $Y=F, \quad U_{Y}=\mathbf{0}, \boldsymbol{V}=\mathbf{0}$ on $Y=1$,

$\mathbf{U}=\left(Y\left(1-\frac{1}{2} Y\right), 0\right)$ on $X=0$,

and

$\overline{\mathrm{Pe}}(\mathbf{U} \cdot \nabla) C=\nabla^{2} C \quad$ for $F \leq Y \leq 1, X>0$,

$(\mathbf{n} \cdot \nabla) C=-\mu C$ on $Y=F, \quad C_{Y}=0$ on $Y=1$,

$C=1$ on $X=0$.

The problem is governed by the geometric parameters $\varepsilon$ and $\lambda=\lambda^{*} / a^{*}$, the Péclet number $\overline{\mathrm{Pe}}=2 U_{0}^{*} a^{*} / D^{*}$ and uptake parameter $\mu=a^{*} \mu^{*}$.

Figure 1 shows the solution domain. We initially solve this problem asymptotically in the limit $\lambda \rightarrow 0$, with $\varepsilon$, $\overline{\mathrm{Pe}}$ and $\mu$ formally of $O(1)$. Below we also consider the limits $\varepsilon \rightarrow 0$ (with a flat wall, Sect. 5) and $\mu=O(1 / \lambda$ ) (with strong uptake over a wavy wall, Sect. 4.2). The asymptotic solution has two primary regions: a core region in $\lambda \ll Y \leq 1$ and a wall region where $Y=O(\lambda)$, as shown in Fig. 1. As $\lambda \rightarrow 0$ the wall appears flat at leading order in the core region. Our aim is to determine the leading-order effective dimensionless uptake parameter $\mu_{\text {eff }}$ in the core problem, defined by

$(\mathbf{n} \cdot \nabla) C=-\mu_{\mathrm{eff}} C$ as $Y \rightarrow 0$,

as a function of $\varepsilon$ and $\overline{\mathrm{Pe}}$ as $\lambda \rightarrow 0$. We now reformulate the problem in each region. 
Fig. 1 The solution domain

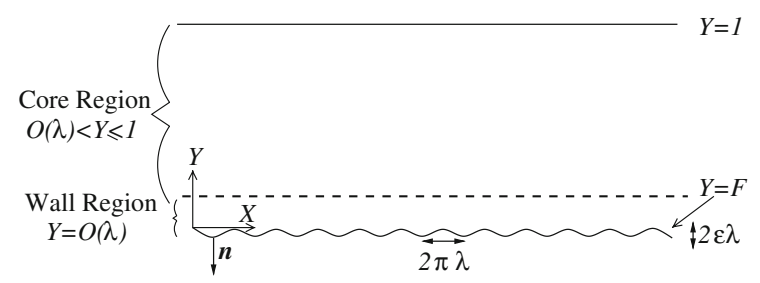

2.1 Core problem: $\lambda \ll Y \leq 1$

Due to the no-slip condition in (2.7b), we expect the flow to approach a shear flow of the form

$\mathbf{U}=(U, V) \sim(Y+\lambda \beta, 0)^{T}$ as $Y \rightarrow 0$,

where $\beta=O(1)$ is a dimensionless slip length to be determined. The leading-order solution of (2.7) in the core region is

$\mathbf{U}=\left(Y\left(1-\frac{1}{2} Y\right), 0\right), \quad P=P_{0}-\frac{1}{2} X$,

for some constant $P_{0}$.

We expand the solute concentration in powers of $Y$ for $\lambda \ll Y \leq 1$ near the wall in terms of unknown coefficients, $C_{i j}(X)$. Equations (2.8) in the core problem then become

$\overline{\mathrm{Pe}}(\mathbf{U} \cdot \nabla) C=\nabla^{2} C$ for $0<Y \leq 1$,

$C \sim C_{00}(X)+C_{01}(X) Y+\lambda\left[C_{10}(X)+C_{11}(X) Y\right]+O\left(\lambda^{2}, Y^{2}\right)$ as $Y \rightarrow 0$,

$C_{Y}=0$ on $Y=1$,

$C=1$ on $X=0, \quad C \rightarrow 0$ as $X \rightarrow \infty$.

The dependence of the coefficients $C_{i j}$ on $X$ is addressed in Sect. 5 below, where we consider the solute transport problem in the core in detail. The dependence on $\mu$ is considered below.

\subsection{Wall problem}

We examine the wall problem near an arbitrary point $X=X_{0}, Y=0$ (where $X_{0} / 2 \pi \lambda$ is an integer) using the scalings

$Y=\lambda(y-\varepsilon), \quad X=X_{0}+\lambda x, \quad U=\lambda u, \quad V=\lambda v$,

with $x, y, u$ and $v$ of $O(1)$ as $\lambda \rightarrow 0$. Since the fully developed flow in the core (2.11) is independent of $x$, we assume $2 \pi$-periodicity of $\mathbf{u}$ in the wall region. The pressure in the core region will vary by $O(\lambda)$ over one wavelength, allowing us to apply $2 \pi$-periodicity to the leading-order pressure field in the wall region. This leaves the local flow problem as

$\nabla_{W} P=\nabla_{W}^{2} \mathbf{u}, \quad \nabla_{W} \cdot \mathbf{u}=0 \quad$ for $y \geq \varepsilon \cos x$,

$\mathbf{u}=\mathbf{0}$ on $y=\varepsilon \cos x, \quad \mathbf{u} \sim(y, 0)+O(1)$ for $1 \ll y \ll 1 / \lambda$,

$\mathbf{u}(x, y)=\mathbf{u}(x+2 \pi, y), \quad P(x, y)=P(x+2 \pi, y)$,

where $\nabla_{W} \equiv(\partial / \partial x, \partial / \partial y)$.

The matching condition on the solute field, $(2.12 \mathrm{~b})$, becomes

$C \sim C_{00}(X)+\lambda\left[C_{01}(X)(y-\varepsilon)+C_{10}(X)\right]+O\left(\lambda^{2}\right)$, 
for $1 \ll y \ll 1 / \lambda$. Although $C$ varies along the channel, we again assume that this variation is small across one wavelength; this assumption is revisited in Sect. 5 below. This allows us to impose $2 \pi$-periodicity of $C$ in the wall problem, treating the coefficients $C_{i j}$ as constants $C_{i j}\left(X_{0}\right)$ in (2.15). Writing $C\left(X_{0}+\lambda x, \lambda y\right)=C_{00}\left(X_{0}\right)+\lambda \dot{c}(x, y)$ and setting $\mathrm{Pe}=\lambda^{2} \overline{\mathrm{Pe}}$, this leaves the wall solute transport problem as

$\operatorname{Pe}\left(\mathbf{u} \cdot \nabla_{W}\right) \dot{c}=\nabla_{W}^{2} \dot{c} \quad$ for $y \geq \varepsilon \cos x$,

$\left(\mathbf{n} \cdot \nabla_{W}\right) \dot{c}=-\mu\left(C_{00}+\lambda \dot{c}\right)$ on $y=\varepsilon \cos x$,

$\dot{c} \sim C_{01}(y-\varepsilon)+C_{10}$ for $1 \ll y \ll 1 / \lambda$,

$\dot{c}(x, y)=\dot{c}(x+2 \pi, y)$,

neglecting terms of $O\left(\lambda^{2}\right)$. For Pe and $\varepsilon$ both fixed, and considering (2.16b), we can then identify two distinct limits: Case 1, where $\mu=O(1)$ and $C_{00}=O(1)$ (which we denote as weak uptake); and Case 2, where $\mu=O(1 / \lambda)$ and $C_{00}=0$ (strong uptake). We now have two well-defined problems for flow and solute uptake in the wall region, which we consider in Sects. 3 and 4, respectively. This will allow us to determine $\mu_{\text {eff }}$ in (2.9) as

$\mu_{\mathrm{eff}}=\frac{C_{01}}{C_{00}}$ in Case $1, \quad \mu_{\mathrm{eff}}=\frac{C_{01}}{\lambda C_{10}}$ in Case 2.

Case 2 also encompasses the limit $\mu \gg 1 / \lambda$.

\subsection{Methods}

For flow and uptake over small-amplitude wavy walls we find asymptotic solutions of (2.14) and (2.16) for $\varepsilon \ll 1$ by expanding variables in powers of $\varepsilon$ and linearising the boundary conditions around $y=0$. This simplifies the governing equations and allows us to construct regular series solutions in $\varepsilon$, in some instances using a simple Crank-Nicolson finite-difference method to compute coefficients.

For walls of finite-amplitude waviness $(\varepsilon=O(1))$ we use the finite-element solver COMSOL Multiphysics to solve for the flow and solute transport (CPU time approximately $1 \mathrm{~min}$ on a desk-top PC). This package discretises the domain into a finite-element mesh of differently sized triangles which are finer near the wavy wall where variation is greatest. To validate the solutions we use mesh refinement, comparison with small-amplitude asymptotics and, for the flow problem, we compare streamlines with Ref. [20] and the predicted slip length with Ref. [6].

\section{Flow in the wall region}

The flow in the wall region is governed by (2.14). We consider first the flow when $\varepsilon \ll 1$. We expand $\mathbf{u}$ and $P$ as a regular power series in $\varepsilon$ about the leading-order shear flow $(y, 0)$. To find the asymptotic solution to $O\left(\varepsilon^{2}\right)$ we Taylor-expand the wall boundary condition around $y=0$ and look at each order separately, writing $\mathbf{u}$ in terms of stream functions. A straightforward calculation gives

$\mathbf{u}=\left(\begin{array}{l}y \\ 0\end{array}\right)+\varepsilon\left(\begin{array}{l}\mathrm{e}^{-y}(y-1) \cos x \\ -y \mathrm{e}^{-y} \sin x\end{array}\right)+\varepsilon^{2}\left(\begin{array}{l}\mathrm{e}^{-y \sqrt{3}}[-\cos y+(\sqrt{3}-1) \sin y] \cos 2 x-1 \\ \frac{1}{2} \mathrm{e}^{-y \sqrt{3}}[\cos y+(\sqrt{3}-4) \sin y] \sin 2 x\end{array}\right)+O\left(\varepsilon^{3}\right)$.

As $y \rightarrow \infty$, (3.1) becomes

$\mathbf{u}=\left(y-\varepsilon^{2}, 0\right)+O\left(\varepsilon^{3}\right)$.

Numerical solutions for finite-amplitude waviness (see Sect. 2.3) are illustrated in Fig. 2. There is no flow reversal for small $\varepsilon$ (Fig. 2a). However, for larger amplitudes, viscous eddies [17] develop in the trough and increase in number for larger amplitudes (see Figs. 2b-d). 
(a)

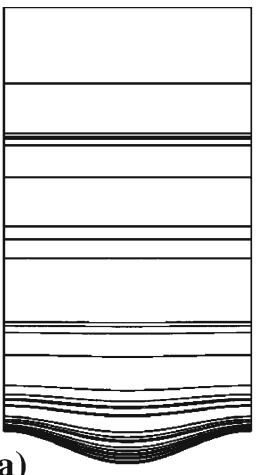

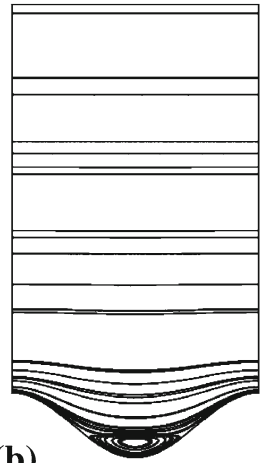

(b)

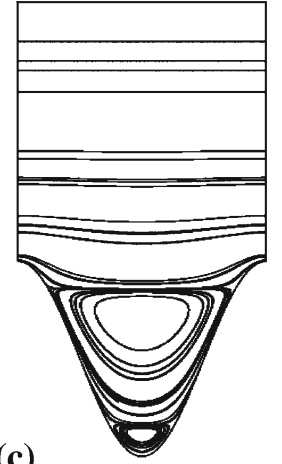

(c)

(d)

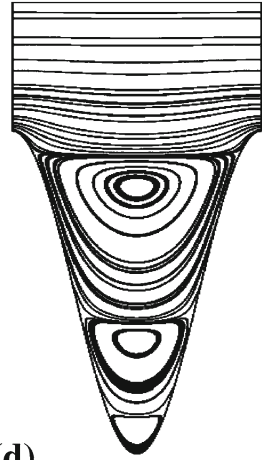

Fig. 2 Streamlines for the flow for $\varepsilon=$ (a) 0.5, (b) 1, (c) 3 and (d) 5 (a) 1

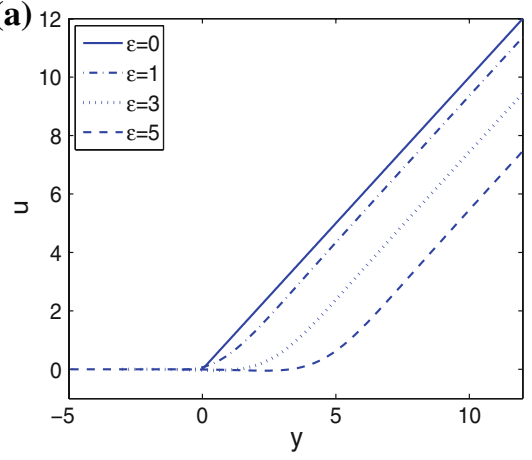

(b) 10

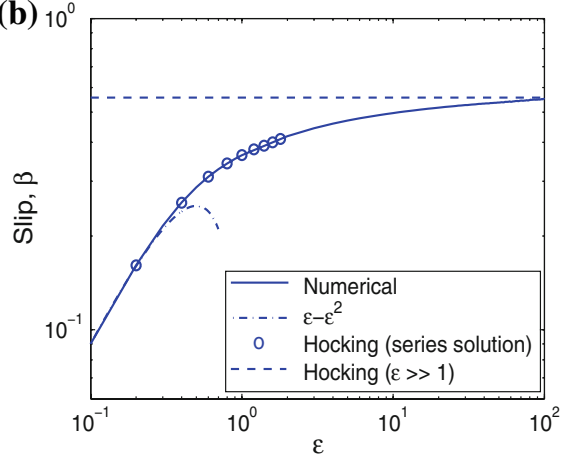

Fig. 3 (a) Velocity $u$ at $x=\pi$ for different values of $\varepsilon$. The crests of the wall lie at $y=\varepsilon$ in each case. (b) Effective slip $\beta$ against amplitude $\varepsilon$ compared with asymptotic and Hocking's (1976) results. The dashed line shows $\beta=\beta_{\infty}$

Figure 3(a) shows the horizontal velocity $u$ at $x=\pi$ for different values of $\varepsilon$. For larger values of $\varepsilon$, the eddies create an area of low velocity in the troughs which decreases the far-field value of $u$. Recall that the crests of the wall lie at $y=\varepsilon$. The far-field value of $\mathbf{u}$ can be represented in terms of the core flow as in (2.10). We plot the slip parameter, $\beta$, in Fig. $3 \mathrm{~b}$ and validate numerical results with (3.2), which predicts $\beta \approx \varepsilon-\varepsilon^{2}$ for $\varepsilon \ll 1$ [6]. $\beta$ increases rapidly until $\varepsilon=O(1)$ and then levels off for larger values of $\varepsilon$. Numerical results agree with Hocking's series-solution slip predictions for moderate $\varepsilon$ (see circles in Fig. 3(b)) and with his prediction for the case $\varepsilon \rightarrow \infty$ $\left(\beta_{\infty} \approx 0.5569\right)$.

\section{Transport in the wall region}

Having determined the flow, we now examine the solute transport. In Sect. 4.1 we study the case $\mu=O(1)$, whilst in Sect. 4.2 we study the case $\mu=O\left(\lambda^{-1}\right)$ which corresponds to stronger uptake. For both cases we discuss small-amplitude asymptotic solutions, numerical solutions at finite amplitude and then match the results with the core solution to find an effective uptake condition via (2.17).

\subsection{Case 1: weak uptake, $\mu=O(1)$}

To leading order in $\lambda$ with $\mu=O(1), \mathrm{Pe}=O(1)$ and $\varepsilon=O(1)$ we solve (2.16) with the prescribed flux condition $\left(\mathbf{n} \cdot \nabla_{W}\right) \dot{c}=-\mu C_{00}$ on $y=\varepsilon \cos x$. To simplify the problem we scale out $\mu$ and simplify the far-field boundary condition by setting $\bar{c}=\left(\dot{c}+\varepsilon C_{01}-C_{10}\right) / \mu C_{00}$, which gives 
$\operatorname{Pe}\left(\mathbf{u} \cdot \nabla_{W}\right) \bar{c}=\nabla_{W}^{2} \bar{c} \quad$ for $y \geq \varepsilon \cos x$,

$\left(\mathbf{n} \cdot \nabla_{W}\right) \bar{c}=-1$ on $y=\varepsilon \cos x$,

$\bar{c} \sim\left(C_{01} / \mu C_{00}\right) y$ for $1 \ll y \ll 1 / \lambda$,

with $\bar{c}(x, y)=\bar{c}(x+2 \pi, y)$. By applying the divergence theorem to (4.1) we find that the flux in the far-field must balance that crossing the wall, and hence that (from (2.17)),

$\frac{C_{01}}{\mu C_{00}}=\frac{\mu_{\mathrm{eff}}}{\mu}=\frac{L(\varepsilon)}{2 \pi}$,

where $L(\varepsilon)$ is the arc-length of the wavy wall over one wavelength.

To solve (4.1) numerically on the finite domain $\varepsilon \cos x \leq y \leq y_{0}$ with $y_{0} \gg 1$, it is convenient to subtract off the far-field concentration using $c=\bar{c}-\frac{C_{01}}{\mu C_{00}} y_{0}$, which gives

$\operatorname{Pe}\left(\mathbf{u} \cdot \nabla_{W}\right) c=c_{x x}+c_{y y}$ for $y \geq \varepsilon \cos x$,

$\left(\mathbf{n} \cdot \nabla_{W}\right) c=-1$ on $y=\varepsilon \cos x, \quad c=0$ on $y=y_{0}$,

$c(x, y)=c(x+2 \pi, y)$.

Appendix A gives details of the small-amplitude solution of (4.1) up to $O\left(\varepsilon^{2}\right)$. The dashed line in Fig. 4(a) shows $c-\left(y-y_{0}\right)$ (the perturbation to the leading-order concentration field from Appendix A) at $x=\pi$ for $\varepsilon=0.1$. Near $y=0$ we see the effect of the flow, where clockwise recirculation of the solute gradient increases and then decreases the concentration perturbation as $y$ increases. The numerical solution for $c-\left(y-y_{0}\right)$ for $\varepsilon=0.1$ compares well with the asymptotic solution up to $O\left(\varepsilon^{2}\right)$. Concentration perturbations are more clearly illustrated in Fig. 5, which shows concentration contours for different amplitudes and Péclet numbers. Figure 4(b) shows the corresponding centreline concentrations. In Fig. 5 we see that for $\mathrm{Pe}=1$ the solute is drawn into the troughs of the wall by diffusion, whereas for $\mathrm{Pe}=100$ the top eddy causes the solute to circulate, making $c(\pi, y)$ non-monotonic in $y$ (Fig. 4b). The lower eddies, however, are too weak to have an effect on the concentration field.

Figure 4(b) also shows that the concentration at the wall is lower for larger amplitudes, increasing the uptake and the slope of $c$ in the far-field. Increasing the Péclet number influences the concentration near the wall but not that in the far-field, consistent with (4.2). Since $L(\varepsilon)>2 \pi$ for all $\varepsilon>0$, solute uptake is always increased by the wavy wall when $\mu=O(1)$.

To illustrate the flux of solute within a trough we plot fluxlines of the incompressible vector field $\mathbf{J}=\operatorname{Pe} c \mathbf{u}-\nabla_{W} c$ for varying Péclet numbers (Fig. 6). The fluxlines indicate the mean direction of particle transport, illustrating competition between advection and diffusion via a random walk. For $\mathrm{Pe}=0$, solute is pulled down through the wavy wall purely by diffusion. As Pe increases we see the effect of the flow on the flux. Figure 6(b) shows that for Pe $=1$, the top eddy causes the solute to circulate, but most of the solute still diffuses through the wall at the bottom of the trough. For $\mathrm{Pe}=100$ (Fig. 6c), the top eddy traps a large quantity of the solute, causing it to re-circulate. The effect of the second eddy is also evident and for larger values of Pe more closed fluxlines form around this eddy.
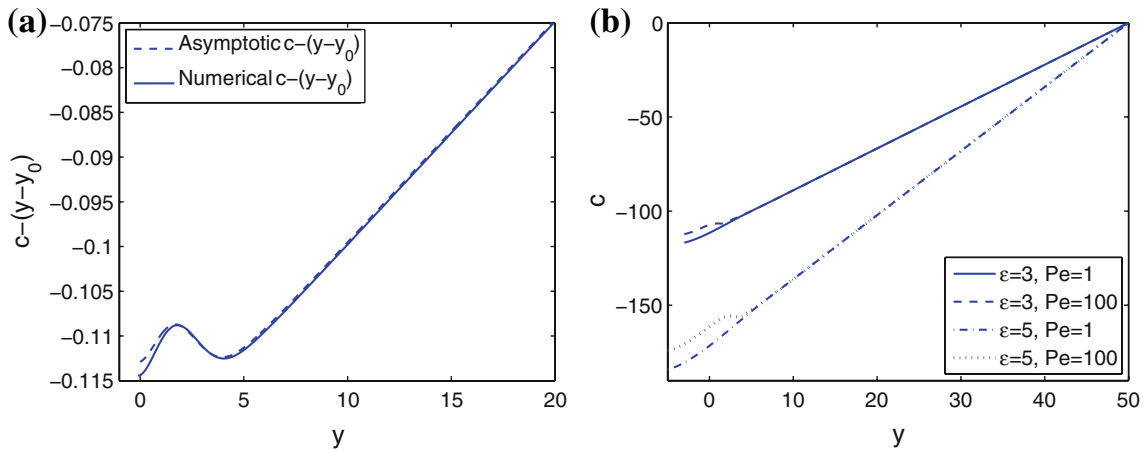

Fig. 4 Case 1: (a) Comparison of asymptotic and numerical predictions of the perturbation to the concentration field $c-\left(y-y_{0}\right)$ at $x=\pi$ for $\mathrm{Pe}=1$ and $\varepsilon=0.1$ (with $y_{0}=50$ ). (b) $c$ at $x=\pi$ for varying Pe and $\varepsilon$ 
(a)

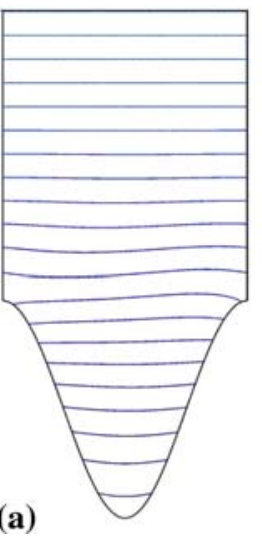

(b)

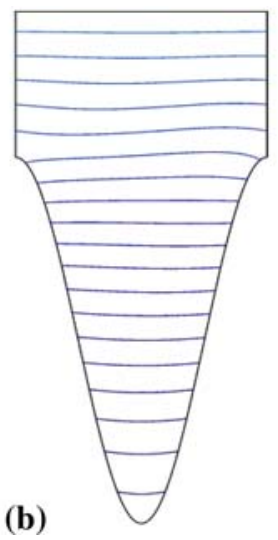

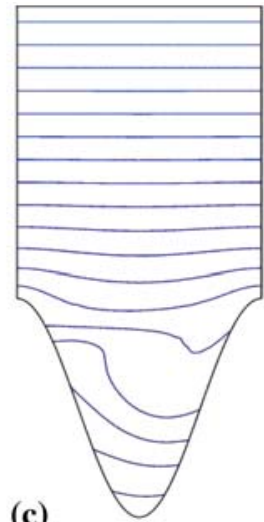

(c)

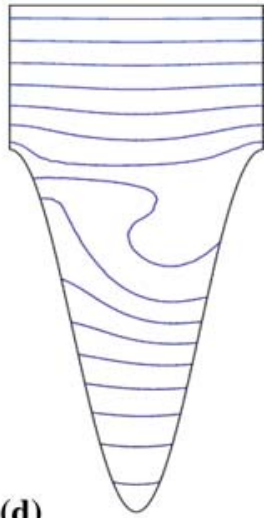

(d)

Fig. 5 Case 1: Equally spaced contour plots showing numerical results for $c$ for $(\mathrm{Pe}, \varepsilon)=(\mathbf{a})(1,3),(\mathbf{b})(1,5),(\mathbf{c})(100,3)$ and $(\mathbf{d})$ $(100,5)$
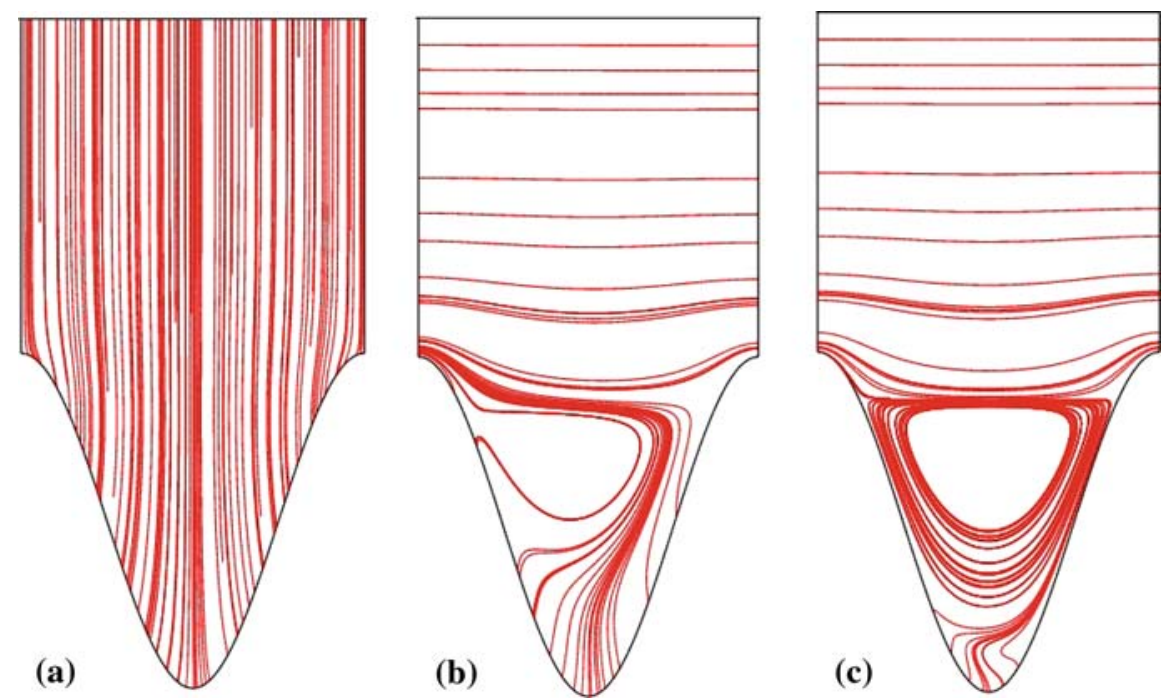

Fig. 6 Case 1: Fluxlines for $\varepsilon=3$ and (a) $\mathrm{Pe}=0$, (b) $\mathrm{Pe}=1$ and (c) $\mathrm{Pe}=100$

\subsection{Case 2: strong uptake, $\mu=O(1 / \lambda)$}

We now take $\bar{\mu} \equiv \lambda \mu=O(1)$. This strong-uptake case leads to low concentration near the wavy wall, so that (2.16b) becomes $\left(\mathbf{n} \cdot \nabla_{W}\right) \dot{c}=-\bar{\mu} \dot{c}$ on $y=\varepsilon \cos x$. To simplify the problem we now define $c=\left(\bar{c} / C_{01}\right)$ to give

$\operatorname{Pe}\left(\mathbf{u} \cdot \nabla_{W}\right) c=\nabla_{W}^{2} c$ for $y \geq \varepsilon \cos x$,

$\left(\mathbf{n} \cdot \nabla_{W}\right) c=-\bar{\mu} c$ on $y=\varepsilon \cos x$,

$c \sim y+\left(\left(C_{10} / C_{01}\right)-\varepsilon\right) \quad$ for $1 \ll y \ll 1 / \lambda$,

with $c(x, y)=c(x+2 \pi, y)$. In this case $\mu_{\mathrm{eff}}=C_{01} / \lambda C_{10}$ must be determined as part of the solution.

To find a numerical solution we again consider the finite domain $\varepsilon \cos x \leq y \leq y_{0}$, replacing the far-field condition in (4.4) with

$c_{y}=1$ on $y=y_{0}$,

and seeking $C_{01} / \lambda C_{10}$ as a function of $\bar{\mu}, \varepsilon$ and Pe. 


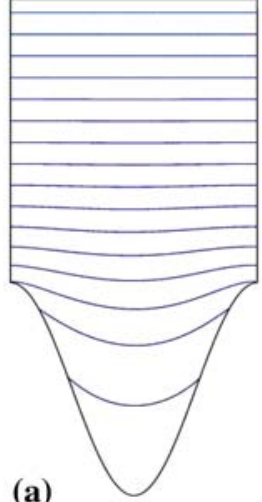

(a)

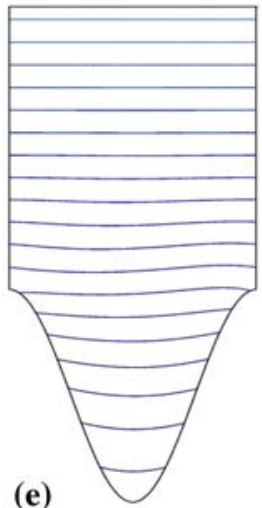

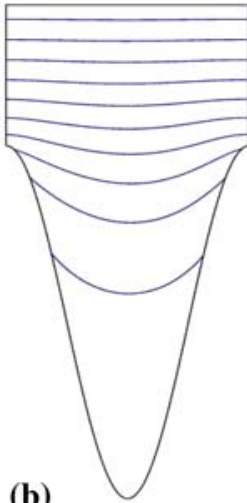

(b)

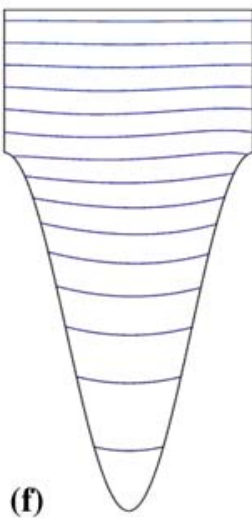

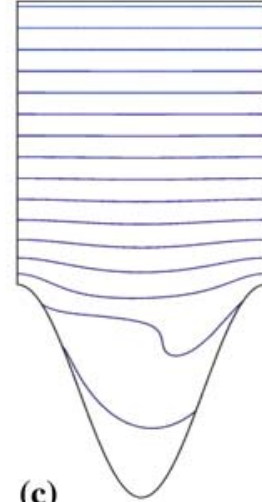

(c)
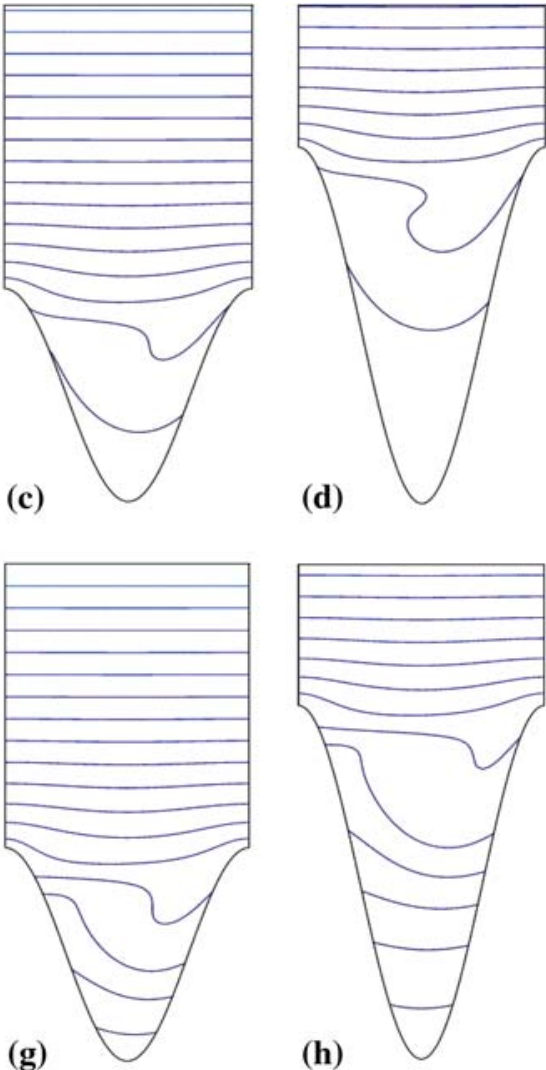

(d)

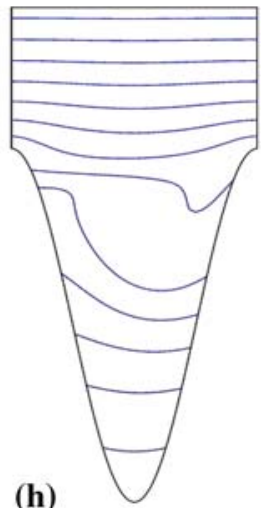

(h)

Fig. 7 Case 2: Equally spaced contour plots showing numerical results for $c$ for $(\bar{\mu}, \mathrm{Pe}, \varepsilon)=(\mathbf{a})(1,1,3),(\mathbf{b})(1,1,5),(\mathbf{c})(1,100,3)$, (d) $(1,100,5),(\mathbf{e})(0.1,1,3),(\mathbf{f})(0.1,1,5),(\mathbf{g})(0.1,100,3)$ and $(\mathbf{h})(0.1,100,5)$

As with Case 1 we first look at the small-amplitude asymptotic solution up to $O\left(\varepsilon^{2}\right.$ ) (for details see Appendix B). For $\varepsilon \ll 1$ we validate the numerical solutions with the asymptotic solution to find good agreement (data not shown). We find that in the far-field (for $y>5$ ) the waviness has little effect as the concentration perturbation flattens to just below zero.

Figure 7(a-d) shows contours of constant concentration for $\varepsilon=O(1), \bar{\mu}=1$ and for various Péclet numbers. For larger amplitudes there is less solute uptake at the bottom of the troughs (where $c$ levels out to zero; see Fig. 8a) as the majority of the solute is absorbed at the peaks. This means that only a small proportion of the surface area is being used for absorption despite the larger surface area. For large Péclet numbers (Fig. 7c, d) the top eddy causes the solute to circulate, promoting transport into the trough (the concentration at $x=\pi$ decreases as both the Péclet number increases and as the amplitude increases; see Fig. 8). For small $\mu$ (Figs. 7e-h, 8b) the solute penetrates further into the trough, as it does in Case 1, since the overall uptake is weaker. For this case we see that the effect of $\mathrm{Pe}$ and $\varepsilon$ is slightly more pronounced, with an increase in either of these variables decreasing the concentration in the trough.

The effective boundary condition (2.17) can be written $\mu_{\text {eff }} \equiv 1 / \lambda A$, where $A \equiv C_{10} / C_{01}$ and $c \sim y+$ $(A-\varepsilon)$ as $y \rightarrow \infty$, from (4.4). For $\varepsilon=0, A=1 / \bar{\mu}$ (for details see Appendix B). Figure 9 shows how $A(\varepsilon, \mathrm{Pe}, \bar{\mu})$ varies with $\bar{\mu}$ and $\varepsilon$ for fixed Pe. For larger values of $\bar{\mu}, A$ increases (and $\mu_{\text {eff }}$ decreases) with $\varepsilon$. As seen in Fig. 7, the strong uptake pulls most of the solute into the peaks, with little solute entering the troughs, so the surface area for absorption is effectively smaller than in the flat wall case. For smaller values of $\bar{\mu}, A$ decreases (and $\mu_{\mathrm{eff}}$ increases) with $\varepsilon$, increasing uptake as the amplitude increases. This is because the uptake has dropped to a level where solute can penetrate the trough and so be absorbed by more of the wall, as seen also in Case 1. Figure 10(a) shows how the effective uptake parameters for Cases 1 and 2 compare well for small $\bar{\mu}$. 

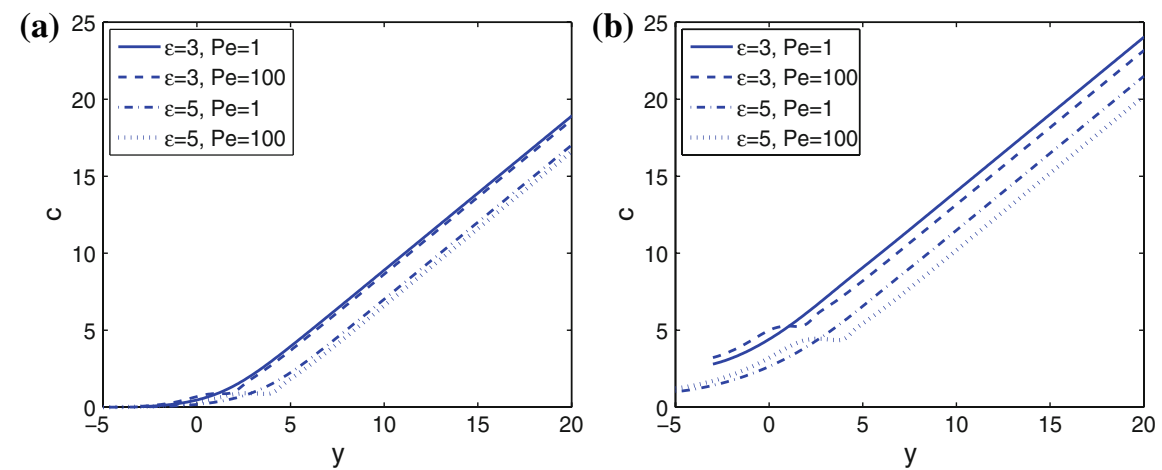

Fig. 8 Case 2: Plots of $c$ at $x=\pi$ for varying Pe and $\varepsilon$ for (a) $\bar{\mu}=1$ and (b) $\bar{\mu}=0.1$, computed with $y_{0}=50$. The crests of the wall lie at $y=\varepsilon$
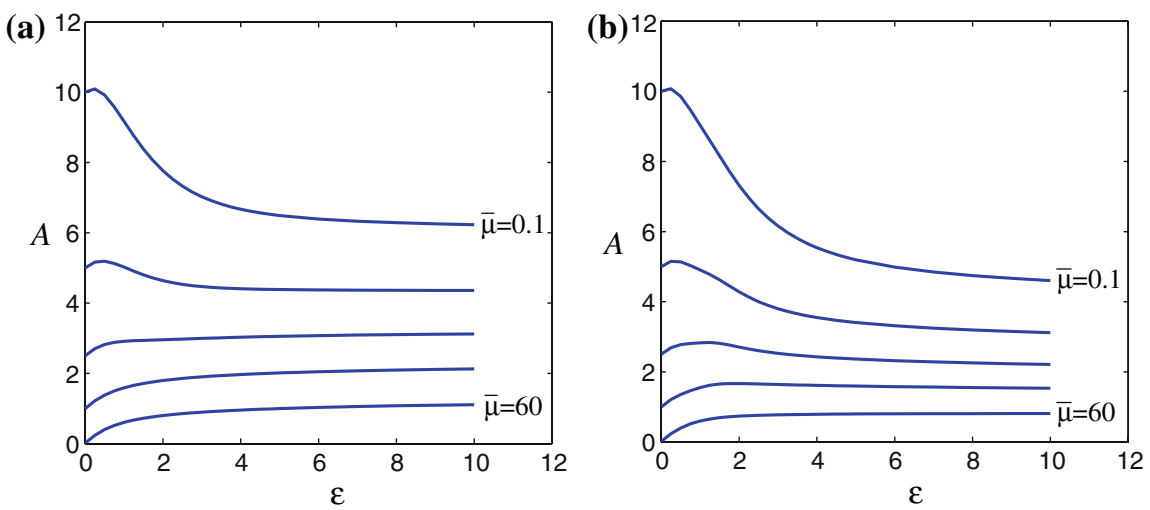

Fig. 9 Case 2: Graphs of $A$ against $\varepsilon$ for $\bar{\mu}=0.1,0.2,0.4,1,60$ and for (a) $\mathrm{Pe}=1$ and (b) $\mathrm{Pe}=100$
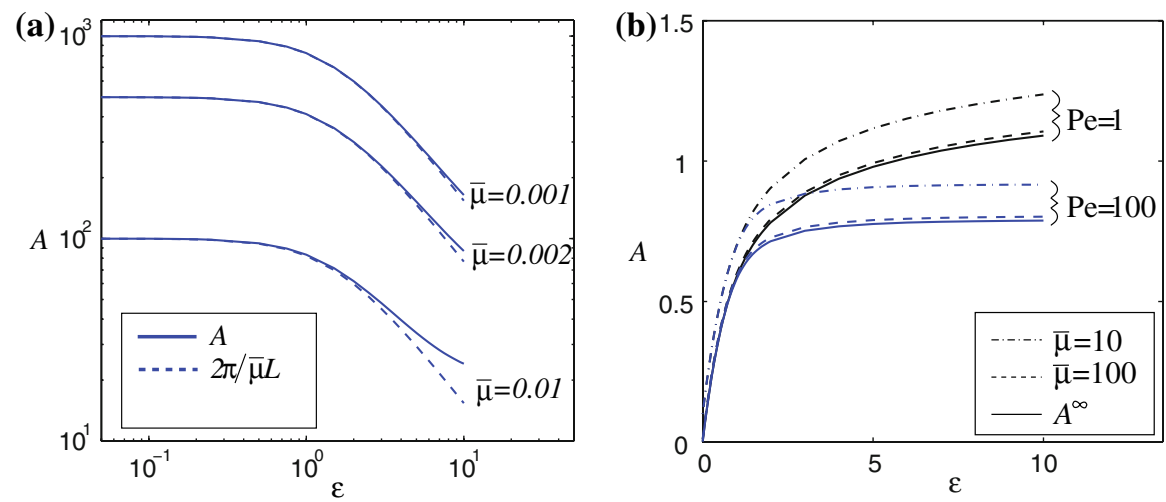

Fig. 10 (a) Comparison of uptake for Case $2\left(1 / \lambda \mu_{\mathrm{eff}}=A\right)$ and Case $1\left(1 / \lambda \mu_{\mathrm{eff}}=2 \pi / \bar{\mu} L\right)$ with $\mathrm{Pe}=1$. (b) Change in $A$ versus $\varepsilon$ as $\bar{\mu} \rightarrow \infty$ with varying Pe

For $\bar{\mu} \gg 1$ and $\varepsilon=0, A=1 / \bar{\mu} \rightarrow 0$. For $\varepsilon>0$, Fig. 10(b) shows $A$ tending to a function independent of $\bar{\mu}$ as $\bar{\mu} \rightarrow \infty$, which we write as $A^{\infty}(\varepsilon, \mathrm{Pe}) . A^{\infty}$ is computed by replacing the uptake condition in (4.4b) with $c=0$ on $y=\varepsilon \cos x . A^{\infty}$ increases monotonically with $\varepsilon$ and falls with Pe, as enhanced mixing increases uptake. Figure 11 also shows that for sufficiently large Pe, $A$ decreases (and $\mu_{\text {eff }}$ increases) for larger values of $\varepsilon$, i.e. mixing by the flow helps carry material deep into the trough. For small $\varepsilon$, however, $A$ is relatively insensitive to Pe. 
Fig. $11 A$ versus $\varepsilon$ for $\bar{\mu}=1$ and varying $\mathrm{Pe}$

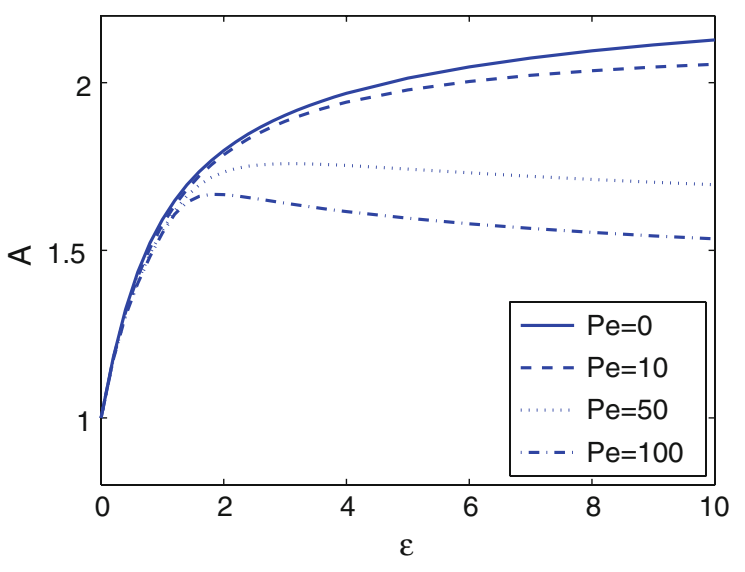

Figure 12(a-c) shows fluxlines for Pe $=0$ and for varying $\bar{\mu}$ : as for Case 1 , diffusion pulls the solute straight down through the wall, but for larger values of $\bar{\mu}$ more solute diffuses through the peaks of the wavy wall, with little reaching the bottom of the trough. Figure $12(\mathrm{~d}-\mathrm{f})$ shows the fluxlines for $\mathrm{Pe}=1$. The flow distorts the fluxlines but does not cause trapping of material. We can see the effect of varying $\bar{\mu}$ in the amount of solute absorbed in each wave of the wall. The ' $x$ 's on the graphs show the flux line that is absorbed at the opposite peak. For larger values of $\bar{\mu}$ this flux line is higher, implying that more solute is absorbed across the cell for larger values of $\bar{\mu}$. For large Péclet numbers (Fig. 12g-i) the flow causes recirculation of the solute around the top eddy, which allows solute to be pulled further into the trough. For larger values of Pe we also see weak recirculation around the second eddy.

A schematic of the fluxlines for large Pe is shown in Fig. 12(j). Solute starting far above the trough is carried by the flow to a downstream cell. All solute that enters the trough is ultimately carried to the wall, although that passing close to the saddle point $S$ may take a long time to do so. The eddy causes trapping of the solute in the troughs, within the homoclinic fluxline through $S$ (recalling of course that fluxlines should be interpreted as providing information about means of distributions of particles undergoing biased random walks).

\section{The core problem over long lengthscales}

We have seen how, for $\mu \ll 1 / \lambda$, the effect of the wall waviness on uptake is to increase $\mu_{\text {eff }}$ by increasing the surface area available for absorption, with the flow in the wall region having little effect ((4.2) and Fig. 10a). For $\mu=O(1 / \lambda)$ or larger, however, we found that increasing wall waviness could reduce $\mu_{\mathrm{eff}}$ (with uptake taking place mainly at the peaks of the wall), although this effect can be offset by flow-induced mixing in the troughs at large Pe (Fig. 10b). We now put these results in context by returning to the core problem (Fig. 1) to examine how the concentration field evolves over distances comparable to, or longer than, the channel width. We also revisit the assumption of Sect. 2.2 that $C$ varies on a lengthscale much longer than $\lambda$.

In the channel the flow is Poiseuille to leading order (see 2.11). We re-express (2.8) and (2.9) as

$\overline{\operatorname{Pe}} Y\left(1-\frac{1}{2} Y\right) C_{X}=C_{X X}+C_{Y Y}$ for $0<Y \leq 1$,

$C_{Y}=\mu_{\mathrm{eff}} C$ on $Y=0, \quad C_{Y}=0$ on $Y=1$,

$C=1$ on $X=0, \quad C \rightarrow 0$ as $X \rightarrow \infty$.

We calculated numerical solutions of (5.1) for $O(1)$ values of the parameters ( $\left.\mu_{\mathrm{eff}}, \overline{\mathrm{Pe}}\right)$ using COMSOL Multiphysics, testing our results against asymptotic predictions derived below. Results are shown in Figs. 13 and 14. As $\mu_{\text {eff }}$ is increased, solute uptake occurs nearer the inlet of the channel so that the concentration along the wall falls much more rapidly than that along the channel centreline (Fig. 15). For large values of $\overline{\mathrm{Pe}}$ and $\mu_{\mathrm{eff}}$, a distinct boundary layer at the channel wall is seen (Fig. 14). We now consider the asymptotic structures underlying these results. 
(a)
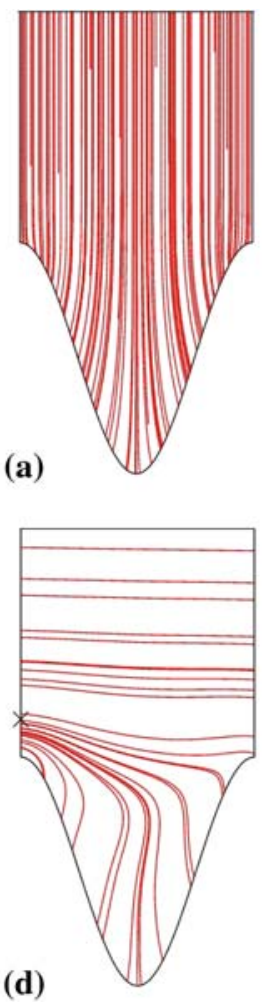

(b)

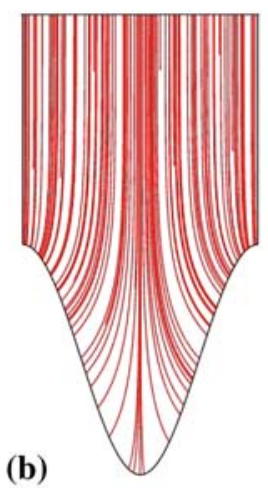

(c)
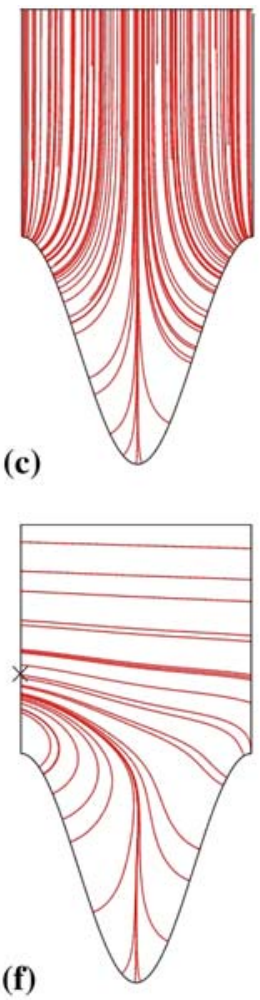

(e)

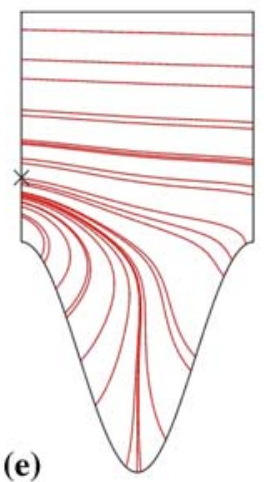

(f)

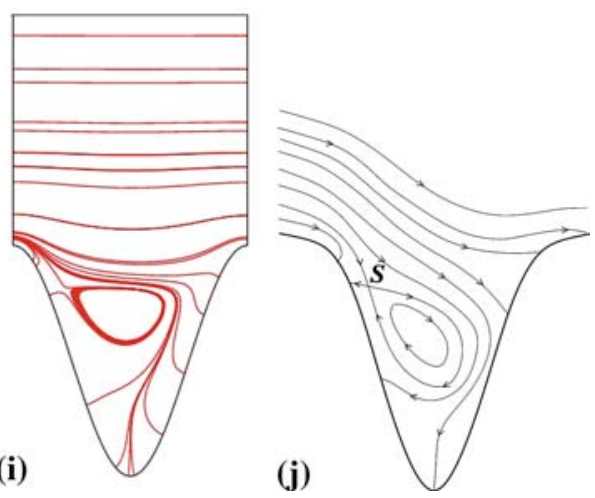

(j) (g)

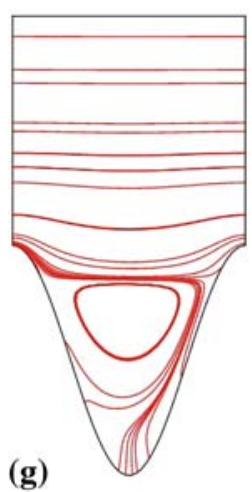

(h)

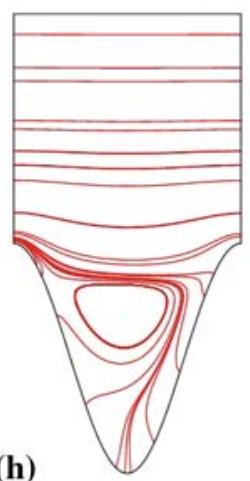

(i)

Fig. 12 Case 2: Fluxlines for $\varepsilon=3$, and $(\bar{\mu}, \mathrm{Pe})=(\mathbf{a})(0.1,0),(\mathbf{b})(1,0),(\mathbf{c})(100,0),(\mathbf{d})(0.1,1),(\mathbf{e})(1,1),(\mathbf{f})(100,1)$, (g) $(0.1,100)$, (h) $(1,100)$, (i) $(100,100)$, and (j) Phase plane diagram for solute transport

\subsection{Asymptotic parameter regimes}

We can split the $\left(\mu_{\mathrm{eff}}, \overline{\mathrm{Pe}}\right)$-parameter space into five distinct regimes $(I-V)$ where asymptotically different solutions can be found; see Fig. 16 (further sub-divisions will be discussed below). These regimes can be characterised by discussing behaviour in the five distinguished limits that separate them.

\subsubsection{The I/II boundary, $\overline{\mathrm{Pe}} \sim \mu_{\mathrm{eff}}^{1 / 2} \ll 1$}

Throughout regimes $I$ and $I I$, rapid transverse diffusion suppresses transverse concentration gradients at leading order (Figs. 13a-b and 14a-b). To describe this, we scale $\overline{\mathrm{Pe}}=\mu_{\mathrm{eff}}^{1 / 2} \Pi$, where $\Pi=O(1)$ on the $I / I I$ boundary and 
(a)

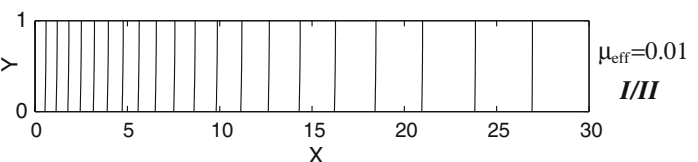

(b)

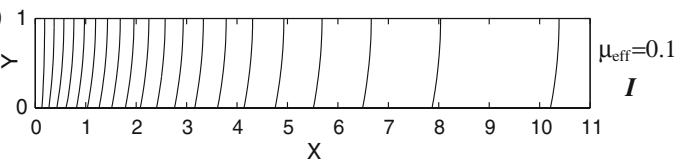

(c)

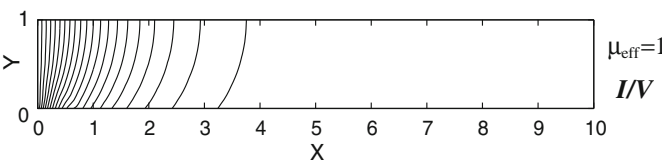

(d)

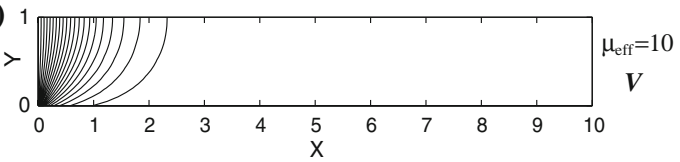

Fig. 13 Equally spaced concentration contours for $\overline{\mathrm{Pe}}=0.1$ and varying $\mu_{\text {eff. Roman numerals denote asymptotic regimes }}$ in Fig. 16 (a)

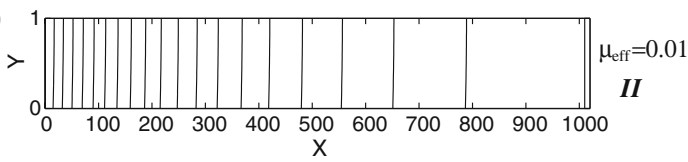

(b)

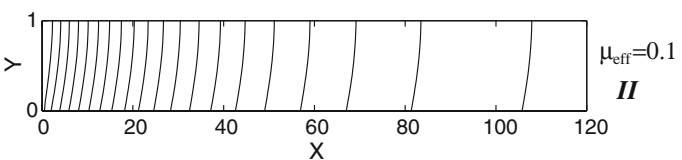

(c)

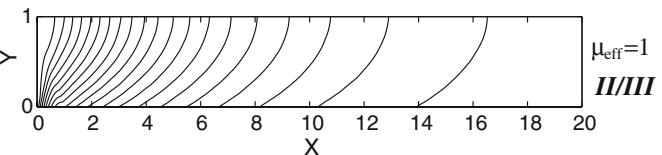

(d)

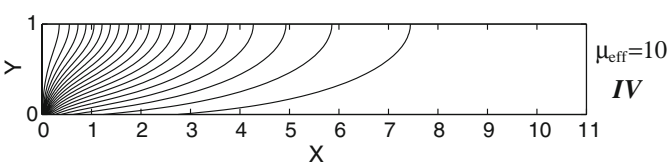

Fig. 14 Equally spaced concentration contours for $\overline{\mathrm{Pe}}=10$

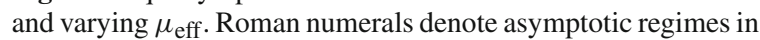
Fig. 16
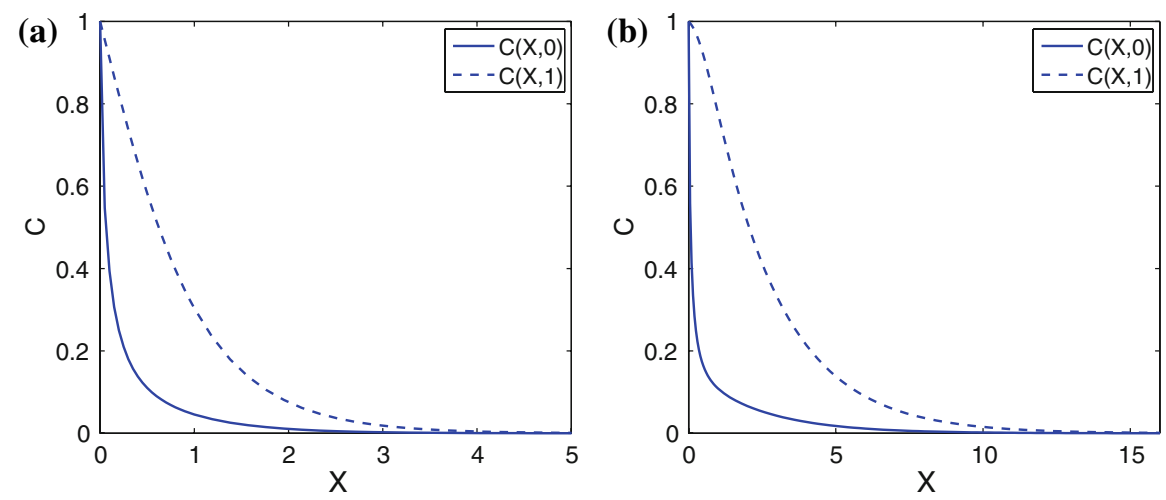

Fig. 15 Graphs of $C$ at $Y=0$ and $Y=1$ with $\mu_{\text {eff }}=10$ for (a) $\overline{\mathrm{Pe}}=0.1$ (regime $V$ ) and (b) $\overline{\mathrm{Pe}}=10$ (regime $I V$ )

$X=\mu_{\mathrm{eff}}^{-1 / 2} \bar{X}$. This converts (5.1) to

$\mu_{\mathrm{eff}} \Pi Y\left(1-\frac{1}{2} Y\right) C_{\bar{X}}=\mu_{\mathrm{eff}} C_{\bar{X} \bar{X}}+C_{Y Y}$,

$C_{Y}=\mu_{\mathrm{eff}} C$ on $Y=0, \quad C_{Y}=0$ on $Y=1$,

$C=1$ on $\bar{X}=0, \quad C \rightarrow 0$ as $\bar{X} \rightarrow \infty$.

We expand $C=C_{0}(\bar{X})+\mu_{\mathrm{eff}} C_{1}(\bar{X}, Y)+O\left(\mu_{\mathrm{eff}}^{2}\right)$, with $C_{0}(0)=1, C_{0} \rightarrow 0$ as $\bar{X} \rightarrow \infty$, which satisfies (5.2) at $O(1)$. At $O\left(\mu_{\mathrm{eff}}\right),(5.2)$ is

П $Y\left(1-\frac{1}{2} Y\right) C_{0 \bar{X}}=C_{0 \bar{X} \bar{X}}+C_{1 Y Y}$,

$C_{1 Y}=C_{0}$ on $Y=0, \quad C_{1 Y}=0$ on $Y=1$,

$C_{1}=0$ on $\bar{X}=0, \quad C_{1} \rightarrow 0$ as $\bar{X} \rightarrow \infty$. 


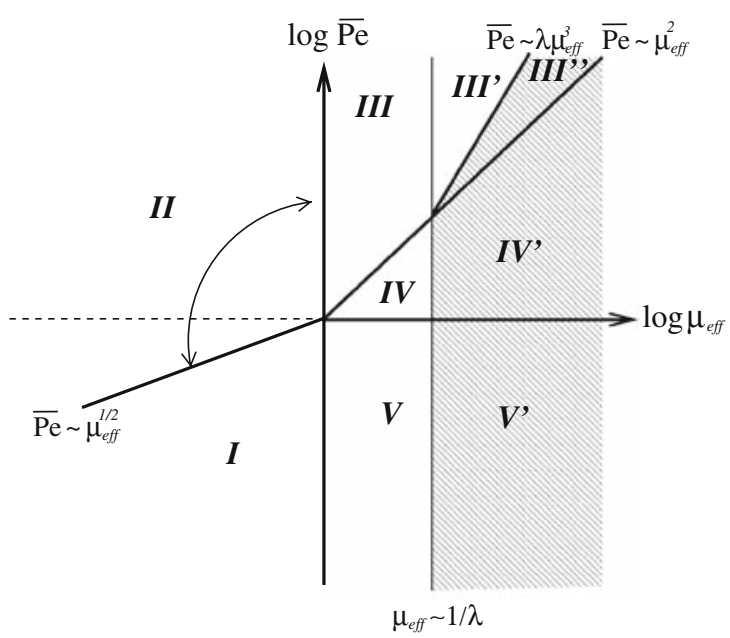

Fig. 16 Asymptotic parameter regimes

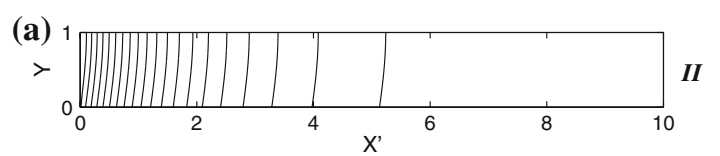

(b)
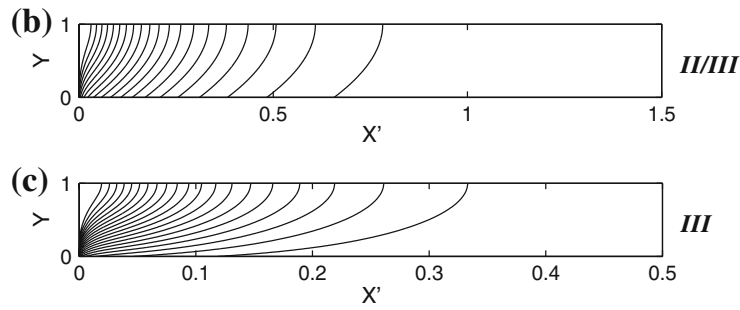

Fig. 17 Boundary of regimes II/III: Equally spaced contour plots of $C$ for (a) $\mu_{\text {eff }}=0.1$, (b) $\mu_{\text {eff }}=1$ and (c) $\mu_{\text {eff }}=10$

We integrate across the channel width to give $\frac{1}{3} \Pi C_{0 \bar{X}}=C_{0 \bar{X}} \bar{X}-C_{0}$, which is easily solved to give

$C_{0}=\exp \left[\frac{1}{6}\left(\Pi-\sqrt{\Pi^{2}+36}\right) \bar{X}\right]$,

consistent with Fig. 13(a). For $\Pi \ll 1$ (regime $I$ ) we obtain at leading order $C=\exp \left[-\mu_{\text {eff }}^{1 / 2} X\right]$, representing a dominant balance between axial diffusion and uptake over long lengthscales (as in Fig. 13b). For $\Pi \gg 1$, the leading-order regime-II solution is (from (5.4))

$C=\exp \left[-3 \mu_{\mathrm{eff}} X / \overline{\mathrm{Pe}}\right]$,

representing a dominant balance between advection and uptake (see [40]). $Y$-dependent corrections to (5.5) remain small over long lengthscales provided $\mu_{\text {eff }} \ll 1$. Equation (5.5) remains a valid description for $\overline{\mathrm{Pe}} \gg 1, \mu_{\text {eff }} \ll 1$, as in Figs. 14(a, b).

\subsubsection{The II/III boundary, $\mu_{\mathrm{eff}}=O(1), \overline{\mathrm{Pe}} \gg 1$}

Motivated by (5.5), and anticipating that transverse concertration gradients become significant for $\overline{\mathrm{Pe}} \gg 1, \mu_{\mathrm{eff}}=$ $O$ (1) (see Fig. 14), we set $X=\overline{\operatorname{Pe}} X^{\prime}$ in (5.1) to give

$Y\left(1-\frac{1}{2} Y\right) C_{X^{\prime}}=\overline{\mathrm{Pe}}^{-2} C_{X^{\prime} X^{\prime}}+C_{Y Y}$,

$C_{Y}=\mu_{\mathrm{eff}} C$ on $Y=0, \quad C_{Y}=0$ on $Y=1$,

$C=1$ on $X^{\prime}=0, \quad C \rightarrow 0$ as $X^{\prime} \rightarrow \infty$.

At leading order we obtain a balance between advection, transverse diffusion and uptake

$Y\left(1-\frac{1}{2} Y\right) C_{X^{\prime}}=C_{Y Y}$,

subject to (5.6b) and (5.6c), which can be solved using a Crank-Nicolson finite-difference scheme, time-stepping in $X^{\prime}$. Solutions are shown in Fig. 17, mirroring the changes seen in Fig. 14(b-d). For small $\mu_{\text {eff }}$ (Fig. 17a), the concentration field is almost uniform across the channel, consistent with the regime- $I I$ description (5.5). Transverse gradients are stronger for $\mu_{\text {eff }}=1$ (Fig. 17b), with rapid axial variation near $X^{\prime}=0$; for large $\mu_{\text {eff }}$ (Fig. 17c) there is rapid uptake of solute at the wall near $X^{\prime}=0$. 


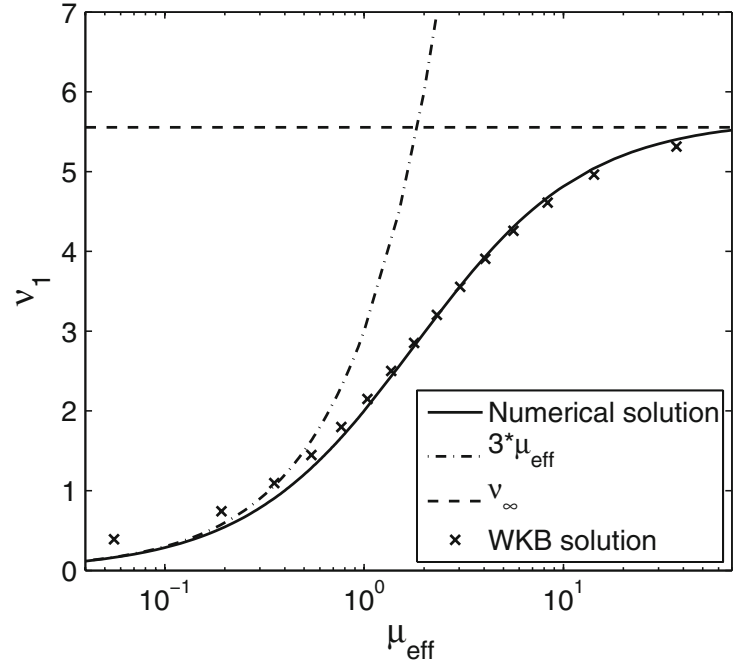

Fig. 18 Boundary of regimes II/III: Eigenvalue $v_{1}$ plotted against $\mu_{\text {eff. Crosses show the WKB solution (C.15) }}$

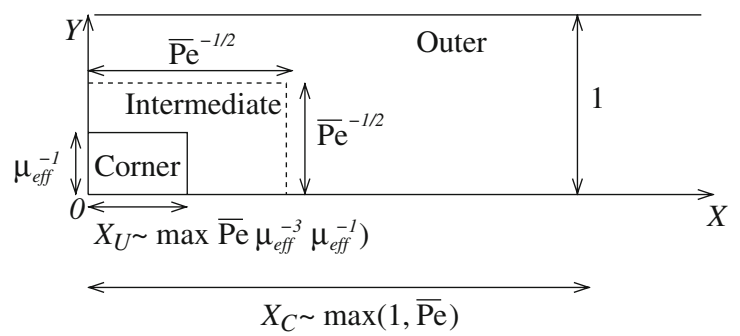

Fig. 19 A schematic illustration of the spatial regions in regimes $I I I, I V$ and $V$

To estimate the rate at which concentration falls along the channel, we express $C$ as a sum of separable eigensolutions

$C=\sum_{n=1}^{\infty} a_{n} \mathrm{e}^{-v_{n} X^{\prime}} F_{n}(Y)$,

where $0<v_{1}<v_{2}<\ldots$, and the eigenfunctions $F_{n}(Y)$ satisfy the orthogonality relation

$\int_{0}^{1} F_{n}(Y) F_{m}(Y) Y\left(1-\frac{1}{2} Y\right) \mathrm{d} Y=\delta_{n m}$.

To satisfy the inlet boundary condition, we must also have

$\sum_{1}^{\infty} a_{n} F_{n}(Y)=1$.

From (5.7), each eigenmode satisfies

$F_{Y Y}+v Y\left(1-\frac{1}{2} Y\right) F=0$,

$F_{Y}=\mu_{\text {eff }} F$ on $Y=0, \quad F_{Y}=0$ on $Y=1$.

The lowest value of $v$, computed numerically, is plotted with a solid line in Fig. 18. For $\mu_{\text {eff }} \ll 1, v_{1} \approx 3 \mu_{\text {eff }}$, consistent with (5.5) in regime II. For $\mu_{\text {eff }} \gg 1$ (regime III), $v_{1}$ approaches a finite value $v_{\infty} \approx 5.555$, which is derived from a WKB approximation (see Appendix C) for higher-order eigenvalues (C.15) and which captures well the lowest-order mode (Fig. 18). The smallest eigenvalue gives only the downstream behaviour in regime III. To find more about the upstream behaviour we look at higher modes, which reveal evidence of a nested boundary layer at the entrance of the channel. This we examine in more detail in the next section.

\subsubsection{Regions III, IV and V}

We provide only a brief overview of regimes $I I I, I V, V$ (Fig. 16), using Fig. 19 to illustrate our argument. Each regime is characterised by an outer region for which $Y=O(1)$ and $C$ varies over a length $X_{C}$, say, and a corner region near $(0,0)$ for which $Y=O\left(\mu_{\text {eff }}^{-1}\right)$ and $C$ varies over a length $X_{U}$. The mixed boundary condition applies 
in (and defines) the corner region, so that $X_{U}$ is the length-scale over which $C$ varies along the wall. The boundary condition $C=0$ applies in the outer region, so that $X_{C}$ defines the lengthscale over which $C$ varies along the channel centreline. Figure 15 (taking data from Figs. 13(d) and 14(d)) shows that $X_{U} \ll X_{C}$ under suitable conditions.

In regime $I I I\left(\overline{\mathrm{Pe}} \gg \mu_{\text {eff }}^{2} \gg 1\right), X_{C}=\overline{\mathrm{Pe}}$ (see Sect. 5.1.2) and $X_{U}=\overline{\mathrm{Pe}} / \mu_{\text {eff }}^{3}$. Both the corner and outer regions are long and thin with a dominant balance between axial advection and transverse diffusion. In the outer region we set $X=\overline{\mathrm{Pe}} X^{\prime}$, which yields (5.7) to leading order. The overlap between the corner and outer regions is then described by the well-known self-similar solution of the problem

$Y C_{X^{\prime}}=C_{Y Y}, \quad C=0$ on $Y=0, \quad C=1$ on $X^{\prime}=0$,

which may be written

$C=\gamma\left(\frac{1}{3}, \frac{1}{9} X^{\prime-1} Y^{3}\right) / \Gamma\left(\frac{1}{3}\right)$,

where $\gamma$ is the incomplete gamma function.

In regime $I V\left(1 \ll \overline{\mathrm{Pe}} \ll \mu_{\mathrm{eff}}^{2}\right), X_{C}=\overline{\mathrm{Pe}}$ and $X_{U}=\mu_{\mathrm{eff}}^{-1}$. The outer problem is as defined in regime $I I I$. For the corner we scale $X=\mu_{\text {eff }}^{-1} \dot{X}, Y=\mu_{\text {eff }}^{-1} \dot{Y}$ which leaves a purely diffusive problem, satisfying to leading order

$C_{\dot{X} \dot{X}}+C_{\dot{Y} \dot{Y}}=0, \quad C_{\dot{Y}}=C$ on $\dot{Y}=0, \quad C=1$ on $\dot{X}=0$.

The far-field limit of (5.14), where $C \approx 0$ on $\dot{Y}=0$, is

$C \sim 2[\arctan (\dot{Y} / \dot{X})] / \pi$ as $|\dot{X}|,|\dot{Y}| \rightarrow \infty$.

A numerical solution of (5.14) and (5.15) is seen in Fig. 20, showing how $C$ decays slowly along the wall. However (5.15) does not correspond to the inner limit of the outer problem (5.13). This transition occurs across a third, intermediate region between the corner and outer regions where axial advection balances diffusion in $X$ and $Y$ over lengthscales $\overline{\mathrm{Pe}}^{-1 / 2}$ in both directions. The inner limit of the intermediate region matches (5.15), whilst the far-field limit matches (5.13). The intermediate region merges with the corner region along the III/IV boundary where $\overline{\mathrm{Pe}} \sim \mu_{\mathrm{eff}}^{2} \gg 1$, and with the outer region along the $I V / V$ boundary where $\overline{\mathrm{Pe}} \sim 1, \mu_{\mathrm{eff}} \gg 1$. The decay of $C$ in the corner region (Fig. 20b) can be compared with Fig. 15(b), which illustrates the different lengthscales over which $C$ varies along $Y=0$ and $Y=1$ in regime IV.

In regime $V\left(\overline{\mathrm{Pe}} \ll 1 \ll \mu_{\mathrm{eff}}\right)$, advection becomes so weak that both the corner and outer regions are purely diffusive at leading order, with $X_{C}=1$ and $X_{U}=\mu_{\text {eff }}^{-1}$. We solve the outer problem in $X \geq 0,0 \leq Y \leq 1$ using a conformal mapping method to give $C=\frac{1}{\pi}\left\{\arctan \left(\frac{\hat{v}+1}{\hat{u}}\right)-\arctan \left(\frac{\hat{v}-1}{\hat{u}}\right)\right\}$, where $\hat{u}=\sinh \left(\frac{\pi}{2} X\right) \cos \left(\frac{\pi}{2}[Y-1]\right)$, $\hat{v}=\cosh \left(\frac{\pi}{2} X\right) \sin \left(\frac{\pi}{2}[Y-1]\right)$. Figure 21 (a) shows the contours of constant concentration for this solution, which can be compared to Fig. 13(d). The corner problem is defined by (5.14) and (5.15). The decay of $C$ in the outer region (Fig. 21b) can be compared to Fig. 15(a), which illustrates the different lengthscales over which $C$ varies along $Y=0$ and $Y=1$.
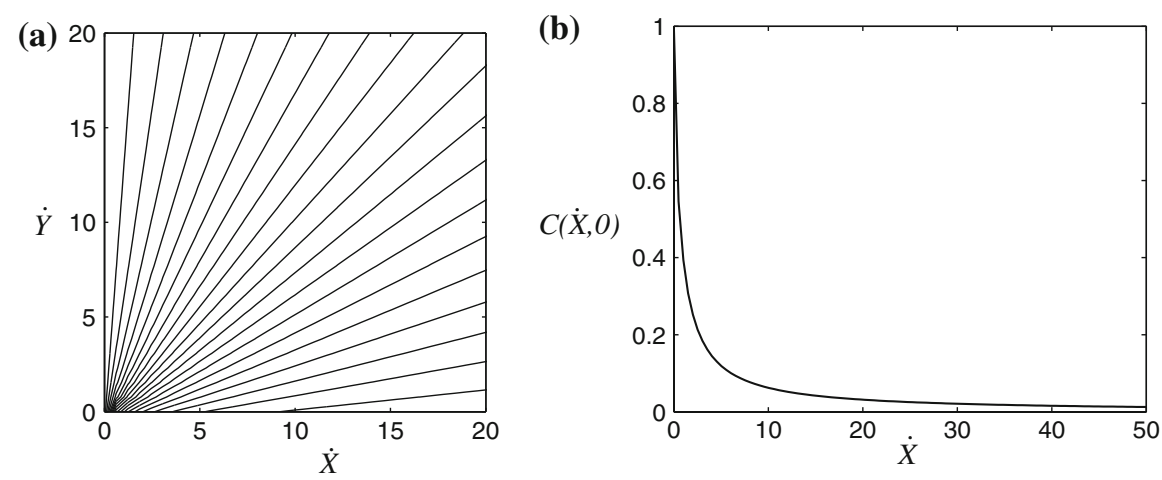

Fig. 20 The corner problem in regime $I V$ satisfying (5.14) and (5.15). (a) Equally spaced contour plot of $C$, (b) Wall concentration $C(\dot{X}, 0)$ versus $\dot{X}$ 

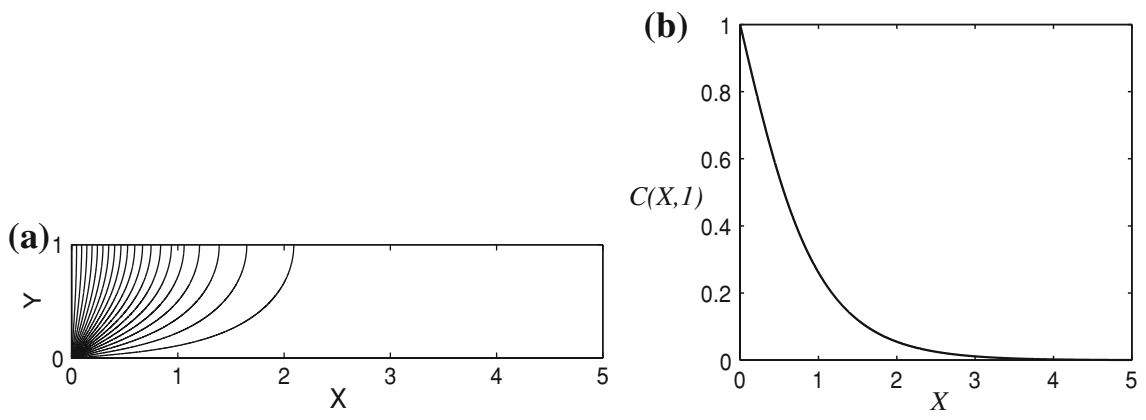

Fig. 21 Outer problem for regime $V$. (a) Equally spaced contour plot of $C$, (b) Plot of $C$ at $Y=1$

On the III/IV boundary, the corner region has $O(1)$ aspect ratio and there is a dominant balance between advection and diffusion in both the axial and transverse direction, with (5.13) again providing overlap with the outer. The same dominant balance appears in the outer region at the overlap between regimes $I V$ and $V$. Finally, on the boundary between regimes $V$ and $I$ with $\mu_{\text {eff }}=O(1), \overline{\mathrm{Pe}} \ll 1$, the corner and outer regions merge.

\subsection{Implications for the wavy wall solution}

We can now revisit the assumption of Sect. 2.2 that $C$ varies on a lengthscale much longer than $\lambda \ll 1$. This applies throughout regimes $I$ and $I I$, and in regimes $I V$ and $V$ for $\mu \sim \mu_{\text {eff }} \ll 1 / \lambda$ (where $X_{U}=\mu_{\text {eff }}^{-1}$ defines the shortest lengthscale over which $C$ varies). In regime $I I I$, we require $X_{U}=\overline{\mathrm{Pe}} \mu_{\mathrm{eff}}^{-3} \gg \lambda$ for our assumption to be valid.

Thus $C$ varies on a lengthscale much longer than $\lambda$ where $\mu_{\text {eff }} \ll \max \left[\left(\overline{\mathrm{Pe}} \lambda^{-1}\right)^{1 / 3}, \lambda^{-1}\right]$ (see Fig. 16). Our assumption is not uniformly valid in regimes $I I I^{\prime \prime}, I V^{\prime}$ and $V^{\prime}$. Case 1 (where $\mu \ll 1 / \lambda$, Sect. 4.1), where increasing wall waviness increases the uptake, applies everywhere else except regime III' Case 2 applies uniformly in regime III', where increasing the waviness decreases uptake.

\section{Extension to a doubly-wavy channel}

The problem described in previous sections deals with the flow and uptake of solute over a sinusoidally wavy wall of wavelength $2 \pi \lambda$ and amplitude $\varepsilon \lambda$, where $\lambda \ll 1$ and $\varepsilon=O(1)$. In this section we extend the discussion to the case of a doubly-wavy wall where the $O(\lambda)$ waviness described previously is corrugated with further sinusoidal waviness of wavelength $2 \pi \lambda^{2}$ and amplitude $\hat{\varepsilon} \lambda^{2}$, where $\lambda \ll 1$ and $\hat{\varepsilon}=O(1)$. In the same way that we divided the previous problem asymptotically into a wall and outer region (Fig. 1), here we divide the domain into three spatial regions (Fig. 22): region A, of width $O(1)$ which fills the bulk of the channel and where we see the doubly-wavy wall as flat along $Y=0$; region $\mathrm{B}$, of width $O(\lambda)$, near the wall where we see the doubly-wavy wall as a single wave along $y=\varepsilon \cos x$; and region $\Gamma$, of width $O\left(\lambda^{2}\right)$ nearest the wall where we only see the $O\left(\lambda^{2}\right)$ waviness.

We formulate the problem separately in each region as before, scaling by a factor $\lambda$ from region $\mathrm{A}$ to region $\mathrm{B}$, and then again with $\lambda$ from region B to region $\Gamma$. First, we define an effective uptake parameter for region $\mathrm{B}, \mu_{\mathrm{effB}}$, which shows the effect of the $O\left(\lambda^{2}\right)$ waviness on the $O(\lambda)$ waviness, such that

$\left(\mathbf{n} \cdot \nabla_{B}\right) C=-\lambda \mu_{\mathrm{effB}} C$ as $y \rightarrow \varepsilon \cos x$,

where $\nabla_{B}$ is the gradient operator expressed using region-B variables. Second, we use this to find an overall effective uptake parameter $\mu_{\text {effA }}$ which shows the effect of both the $O(\lambda)$ and the $O\left(\lambda^{2}\right)$ waviness to give the effective boundary condition for region A

$(\mathbf{n} \cdot \nabla) C=-\mu_{\mathrm{effA}} C$ as $Y \rightarrow 0$. 
Fig. 22 Figure showing the doubly-wavy wall and asymptotic regions $\mathrm{A}, \mathrm{B}, \Gamma$

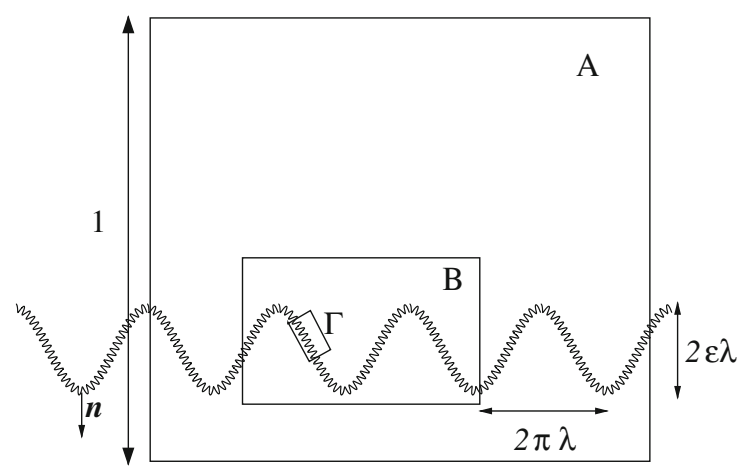

We examine the region- $\Gamma$ problem in the neighbourhood of a point $x=x_{0}, y=\varepsilon \cos x_{0}$ within region $\mathrm{B}$, where the local wall shear rate is $G_{0}\left(x_{0}\right)$. On the $O\left(\lambda^{2}\right)$ lengthscale of region $\Gamma$, the uptake condition becomes

$\left(\mathbf{n} \cdot \nabla_{\Gamma}\right) C=-\lambda^{2} \mu C$,

where $\nabla_{\Gamma}$ is the gradient operator in region- $\Gamma$ variables. From Sect. 4, we may write

$\mu_{\mathrm{effB}}= \begin{cases}\frac{\mu L(\hat{\varepsilon})}{2 \pi} & \text { for } \mu=o\left(\lambda^{-2}\right), \\ \frac{1}{\lambda^{2} A\left(\hat{\varepsilon}, \lambda^{2} \widehat{\mathrm{Pe}}, \lambda^{2} \mu\right)} & \text { for } \mu=O\left(\lambda^{-2}\right),\end{cases}$

where $L(\hat{\varepsilon})$ is the arc-length of the wavy wall over one wavelength in region $\Gamma$ and $\widehat{\mathrm{Pe}}=G_{0}\left(x_{0}\right) \mathrm{Pe}$ is the region- $\Gamma$ Péclet number, dependent on the shear at $x_{0}$. Recall that $A\left(\hat{\varepsilon}, \lambda^{2} \widehat{\mathrm{Pe}}, \lambda^{2} \mu\right) \rightarrow 2 \pi / \lambda^{2} \mu L(\hat{\varepsilon})$ as $\lambda^{2} \mu \rightarrow 0$ and $A\left(\hat{\varepsilon}, \lambda^{2} \widehat{\mathrm{Pe}}, \lambda^{2} \mu\right) \rightarrow A^{\infty}\left(\hat{\varepsilon}, \lambda^{2} \widehat{\mathrm{Pe}}\right)$ as $\lambda^{2} \mu \rightarrow \infty$ (Fig. 10a,b).

We assume that the effect of the slip on region B from region $\Gamma$ is negligible, so the flow in region $\mathrm{B}$ is governed by the same equations seen in the previous wall problem (see Sect. 3). The effective boundary condition in region A can be extracted from (6.4), to give

$\mu_{\mathrm{effA}}= \begin{cases}\frac{\mu L(\hat{\varepsilon}) L(\varepsilon)}{4 \pi^{2}} & \text { for } \mu=o\left(\lambda^{-1}\right), \\ \frac{1}{\lambda A(\varepsilon, \mathrm{Pe}, \lambda \mu L(\hat{\varepsilon}) / 2 \pi)} & \text { for } \mu=O\left(\lambda^{-1}\right), \\ \frac{1}{\lambda A^{\infty}(\varepsilon, \mathrm{Pe})} & \text { for } \mu \gg \lambda^{-1} .\end{cases}$

Note that $\mu_{\text {effA }}$ is independent of $\widehat{P e}$ for all values of $\mu$.

For weak uptake $\left(\mu=o\left(\lambda^{-1}\right)\right)$ the effective uptake parameter for region A is dependent solely on the total surface area available. For stronger uptake $\left(\mu=O\left(\lambda^{-1}\right)\right) \mu_{\text {effA }}$ is governed by the function $A$, but with the original uptake parameter $\mu$ replaced by $\mu L(\hat{\varepsilon}) / 2 \pi \geq \mu$. Thus uptake increases with $\varepsilon$ for small values of $\lambda \mu L(\hat{\varepsilon}) / 2 \pi$ (Fig. 9), but decreases with $\varepsilon$ for larger values of $\lambda \mu L(\hat{\varepsilon}) / 2 \pi$. In particular corrugations at the finest scale have no effect on the overall uptake when the diffusive lengthscale $1 / \mu$ is much smaller than the largest corrugation wavelength $\lambda$.

\section{Discussion}

Many biological surfaces, such as small intestines and blood vessels, use highly ordered hierarchical structures to regulate solute permeation. For oral drug delivery and nutrient absorption, it is known that villi and microvilli on the intestine wall have significant effects on solute permeation and absorption. Many studies have been focused on 
in vitro and in vivo measurements of solute permeation of small intestines [41-44]. Attempts have been also made to predict drug absorption [45]. However, only a few studies have addressed the effect of the hierarchical structures of villi and microvilli on solute absorption [46]. In this paper we have examined the effect of such multi-scale wavy structures on flow and solute absorption via a model problem, involving 2D Stokes flow in a wavy-walled channel, where the amplitude and wavelength of the waviness are both small compared to the channel width. The domain has two primary asymptotic regions: a core region of width $O(1)$ and a region near the wavy wall of width $O(\lambda)$. By solving the transport problem in the wall region numerically, validating our results against asymptotic solutions at small amplitude, we showed how the effective uptake parameter in the core region $\mu_{\text {eff }}$ can be related to the wall uptake parameter $\mu$, as a function of wall shape and Péclet number. This complements the effective slip condition that must be applied to the core flow as a result of wall waviness.

Uptake in the wall region exhibits two distinct limits. When $\mu \ll \lambda^{-1}$, the uptake is sufficiently weak for the concentration not to vary significantly across the wall region. In this case the strength of the flux across the wall is determined by two factors: the local concentration in the core region, and the surface area of the wall. While the net flux is independent of Péclet number, fluxline plots (Fig. 6) show that advection affects the detailed pattern of uptake within each wave of the wall. In particular, we identified a saddle point and closed streamlines in the fluxline distribution, indicating individual particles may experience long transit times (on average) before they reach the wall.

When $\mu=O\left(\lambda^{-1}\right)$, the uptake is strong enough for the concentration in the wall region to fall significantly below that in the core. The net uptake is now dependent on the local flow conditions and wall geometry. Uptake again increases with surface area (arising from increasing the amplitude parameter $\varepsilon$ ) provided $\lambda \mu$ is sufficiently small. However, for larger $\lambda \mu$, uptake diminishes with increasing $\varepsilon$. This is because strong uptake causes the solute to be strongly absorbed at the peaks of the wall, with little solute entering the troughs. Increasing $\varepsilon$ narrows the peaks and reduces their exposure to solute. Fluxlines again demonstrate how the flow may trap particles over long periods (Fig. 12).

Having obtained a local understanding of the effect of near-wall flow on uptake, we then determined the solute distribution in the channel as a whole, when solute is realeased from a distributed source of fixed concentration at the upstream end of the channel. Guided by numerical simulations, we identified five primary parameter regimes where different behaviour occurs (Fig. 16). When uptake is very weak, the concentration distribution extends over long regions down the channel, with diffusion suppressing transverse gradients. As the longest lengthscale over which $C$ varies is independent of $\mu_{\text {eff }}$ in regimes $I I I, I V$ and $V$, we see that waviness shortens the distance over which the solute is absorbed along the channel only in regimes $I$ and II. For stronger uptake, solute concentration falls rapidly along the wall near the source; strong advection can nevertheless sweep solute significant distances down the channel, leading to strong transverse concentration gradients. We used this analysis to identify parameter regimes in which it is appropriate to make the assumption that the concentration varies over a lengthscale much longer than $\lambda$, ensuring that the approximation for $\mu_{\text {eff }}$ is uniformly valid.

We also considered multiple scales of waviness by examining the problem of a doubly-wavy channel, with waves of wavelength $\lambda$ in a channel of width $O(1)$ having corrugations of wavelength $O\left(\lambda^{2}\right)$. We found that for $\mu \ll \lambda^{-1}$ the effective uptake is proportional to the total surface area, while for $\mu=O\left(\lambda^{-1}\right)$ the effective uptake is determined by the flow near the larger wave but is still influenced by the enhanced surface area of the smaller wave. For larger $\mu$ the effective uptake is independent of the smaller scale of waviness.

In the small intestine the Péclet number is large [46] and the uptake parameter $\mu$ can be $O(1)$ [40]. Stoll et al. [46] report $\mu^{*}=0.1 \mathrm{~cm}^{-1}$ for insulin, for example, giving $\mu=0.2$ assuming $a^{*}=2 \mathrm{~cm}$. However, $\mu$ is likely to vary dependent on the solute used. Hence transport in the small intestine is likely to span regions $I I$ and $I I I$ of parameter space (see Fig. 16). This implies that both villi and microvilli in the small intestine increase the uptake proportionally to their surface area. We hope to investigate the application of this work to the small intestine in more detail in a further paper.

These results have a number of significant limitations and some obvious extensions. We did not consider very large values of $\varepsilon$, which in the case of weak uptake $\mu=O\left(\lambda^{-1}\right)$ might increase uptake sufficiently to violate the assumption of small concentration variations across the wall region. We did not explore conditions in which 
uptake is sufficiently strong that the concentration falls appreciably across a single wave (the shaded area in Fig. 16). We assumed a rigid wall, although one can anticipate significant fluid-structure interaction in many biological applications [47] which would have a leading-order effect on uptake. Finally, this study is restricted to two spatial dimensions, and extensions to the three-dimensional geometries that arise in applications such as the small intestine are likely to reveal significantly different flow structures and uptake patterns.

Acknowledgements This work is supported by an EPSRC industrial CASE award with Unilever, made available through the Knowledge Transfer Network for Industrial Mathematics.

Open Access This article is distributed under the terms of the Creative Commons Attribution Noncommercial License which permits any noncommercial use, distribution, and reproduction in any medium, provided the original author(s) and source are credited.

\section{Appendix A: Small-amplitude asymptotic solution for Case 1}

We consider the solution of (4.1) when the wall has small amplitude. For $\varepsilon \ll 1$ we expand $\bar{c}=\bar{c}_{0}+\varepsilon \bar{c}_{1}+\varepsilon^{2} \bar{c}_{2}+$ $O\left(\varepsilon^{3}\right), \frac{C_{01}}{\mu C_{00}}=r_{0}+\varepsilon r_{1}+\varepsilon^{2} r_{2}+O\left(\varepsilon^{3}\right)$ and expand the outward pointing normal as

$\mathbf{n}=\left(-\varepsilon \sin x,-1+\frac{1}{2} \varepsilon^{2} \sin ^{2} x\right)+O\left(\varepsilon^{3}\right)$.

We Taylor-expand the boundary condition on the wall around $y=0$ and use the asymptotic solution for $\mathbf{u}$ given in Sect. 3. At $O(1)$ the wall is effectively flat and $\mathbf{u}$ is a uniform shear slow yielding $\bar{c}_{0}=y$, and $r_{0}=1$.

At $O(\varepsilon),(4.1)$ and $(3.1)$ give

Pe $y \bar{c}_{1 x}-\bar{c}_{1 x x}-\bar{c}_{1 y y}=\operatorname{Pe} y \mathrm{e}^{-y} \sin x$,

$\bar{c}_{1 y}=0$ on $y=0, \quad \bar{c}_{1} \rightarrow r_{1} y$ as $y \rightarrow \infty$,

$\bar{c}_{1}(x, y)=\bar{c}_{1}(x+2 \pi, y)$.

We solve this numerically, first truncating the domain at $y=y_{0} \gg 1$ in the far field and subtracting off the far-field concentration using $c_{1}=\bar{c}_{1}-r_{1} y_{0}$. We then set $c_{1}(x, y)=a(y) \cos x+b(y) \sin x$ to convert (A.2) to a set of coupled ODEs in $a$ and $b$ :

Pe $y b+a-a_{y y}=0$,

- Pe $y a+b-b_{y y}=$ Pe $y \mathrm{e}^{-y}$,

with $a_{y}, b_{y}=0$ on $y=0$ and $a, b=0$ on $y=y_{0}$. We solve (A.3) numerically using a simple finite-difference scheme in $y$.

Likewise at $O\left(\varepsilon^{2}\right)$,

$\operatorname{Pe} y \bar{c}_{2 x}-\bar{c}_{2 x x}-\bar{c}_{2 y y}=-\frac{1}{2} \operatorname{Pe}^{-y \sqrt{3}}[\cos y(\sqrt{3}-4) \sin y] \sin 2 x+\operatorname{Pe}^{-y}\left[y \sin x \bar{c}_{1 y}-(y-1) \cos x \bar{c}_{1 x}\right]$,

with $\bar{c}_{2 y}=-\sin x \bar{c}_{1 x}-\cos x \bar{c}_{1 y y}+\frac{1}{2} \sin ^{2} x$ on $y=0, \bar{c}_{2}=r_{2} y$ as $y \rightarrow \infty$ and $\bar{c}_{2}(x, y)=\bar{c}_{2}(x+2 \pi, y)$. Again we solve this numerically on the truncated domain using $c_{2}=\bar{c}_{2}-r_{2} y_{0}$. We then expand $c_{2}$ as $c_{2}(x, y)=$ $d(y) \cos 2 x+e(y) \sin 2 x+f(y)$, which leaves (A.4) as

$\frac{1}{2} \mathrm{Pe} y e+\frac{1}{4} d-d_{y y}=-\frac{1}{2} \operatorname{Pe}^{-y}\left[(y-1) b+y b_{y}\right]$,

$-\frac{1}{2} \operatorname{Pe} y d+\frac{1}{4} e-e_{y y}=-\frac{1}{2} \operatorname{Pe} \mu \mathrm{e}^{-y \sqrt{3}}[\cos y(\sqrt{3}-4) \sin y]+\frac{1}{2} \operatorname{Pe}^{-y}\left[(y-1) a+y a_{y}\right]$,

$f_{y y}=\frac{1}{2} \operatorname{Pe}^{-y}\left[(y-1) b-y b_{y}\right]$,

with $d_{y}=-\frac{1}{2} a-\frac{1}{2} a_{y y}-\frac{1}{4}, e_{y}=-\frac{1}{2} b-\frac{1}{2} b_{y y}, f_{y}=\frac{1}{2} a-\frac{1}{2} a_{y y}+\frac{1}{4}$, on $y=0$ and $d, e, f=0$, on $y=y_{0}$. Again we solve this numerically using a simple finite-difference scheme. To compare the asymptotic solution to later numerical solutions, we truncate the domain and set $c_{0}=\bar{c}_{0}-r_{0} y_{0}=y-y_{0}$. 


\section{Appendix B: Small-amplitude asymptotic solution for Case 2}

Here we consider solutions of (4.4) for $\varepsilon \ll 1$. We again expand $c=c_{0}+\varepsilon c_{1}+\varepsilon^{2} c_{2}+O\left(\varepsilon^{3}\right)$ and set $\frac{C_{10}}{C_{01}}=$ $s_{0}+\varepsilon s_{1}+\varepsilon^{2} s_{2}+O\left(\varepsilon^{3}\right)$. Using the asymptotic solution of $\mathbf{u}$ from (3.1) and assuming no $x$-dependence of $c_{0},(4.4)$ reduces at leading order to $c_{0 y y}=0$, with $c_{0 y}-\bar{\mu} c_{0}=0$ on $y=0$ and $c_{0} \rightarrow y+s_{0}$ as $y \rightarrow \infty$, which we solve to give

$c_{0}=y+1 / \bar{\mu}$,

and $s_{0}=1 / \bar{\mu}$. The $O(\varepsilon)$ problem for $c_{1}$ is

Pe $y c_{1 x}-c_{1 x x}-c_{1 y y}=\operatorname{Pe} y \mathrm{e}^{-y} \sin x$,

$c_{1 y}-\bar{\mu} c_{1}=\bar{\mu} \cos x$ on $y=0, \quad c_{1}=y+s_{1}-1$ as $y \rightarrow \infty$,

$c_{1}(x, y)=c_{1}(x+2 \pi, y)$.

We simplify this using the Fourier series $c_{1}(x, y)=h(y) \cos x+j(y) \sin x$, to give

$\operatorname{Pe} y j+h-h_{y y}=0, \quad-\operatorname{Pe} y h+j-j_{y y}=\operatorname{Pe} y \mathrm{e}^{-y}$,

with $h_{y}-\bar{\mu} h=\bar{\mu}, j_{y}-\bar{\mu} j=0$ on $y=0, h_{y}, j_{y}=0$ as $y \rightarrow \infty$, which we solve numerically. Here the far-field boundary condition has been simplified to $c_{1 y} \rightarrow 1$.

The $O\left(\varepsilon^{2}\right)$ equation is

$\operatorname{Pe} y c_{2 x}-c_{2 x x}-c_{2 y y}=-\frac{1}{2} \operatorname{Pe}^{-y \sqrt{3}}[\cos y(\sqrt{3}-4) \sin y] \sin 2 x+\operatorname{Pe}^{-y}\left[y \sin x c_{1 y}-(y-1) \cos x c_{1 x}\right]$,

with $c_{2 y}-\bar{\mu} c_{2}=-\frac{1}{4} \cos 2 x+\frac{1}{4}-\sin x c_{1 x}+\bar{\mu} \cos x c_{1 y}-\cos x c_{1 y y}$ on $y=0, c_{2} \rightarrow y+s_{2}$ as $y \rightarrow \infty$ and $c_{2}(x, y)=c_{2}(x+2 \pi, y)$. We again simplify the far-field boundary condition to $c_{2 y} \rightarrow 1$ and use a Fourier series, where $c_{2}(x, y)=k(y) \cos 2 x+m(y) \sin 2 x+n(y)$, to give

$\frac{1}{2} \mathrm{Pe} y m+\frac{1}{4} k-k_{y y}=-\frac{1}{2} \operatorname{Pe}^{-y}\left[(y-1) j+y j_{y}\right]$,

$-\frac{1}{2} \operatorname{Pe} y k+\frac{1}{4} m-m_{y y}=-\frac{1}{2} \operatorname{Pe}^{-y \sqrt{3}}[\cos y(\sqrt{3}-4) \sin y]+\frac{1}{2} \operatorname{Pe}^{-y}\left[(y-1) h+y h_{y}\right]$,

$n_{y y}=\frac{1}{2} \operatorname{Pe}^{-y}\left[(y-1) j-y j_{y}\right]$,

$k_{y}-\bar{\mu} k=-\frac{1}{2} h+\frac{1}{2} \bar{\mu} h_{y}-\frac{1}{2} h_{y y}-\frac{1}{4}$, on $y=0$,

$m_{y}-\bar{\mu} m=-\frac{1}{2} j+\frac{1}{2} \bar{\mu} j_{y}-\frac{1}{2} j_{y y}$, on $y=0$,

$n_{y}-\bar{\mu} n=\frac{1}{2} h+\frac{1}{2} \bar{\mu} h_{y}-\frac{1}{2} h_{y y}+\frac{1}{4}$, on $y=0$,

$k_{y}, m_{y}, n_{y}=0$, as $y \rightarrow \infty$.

We solve (B.5) using a finite-difference scheme. 


\section{Appendix C: WKB method for the outer problem where $\mu_{\text {eff }}=O(1), \overline{\mathrm{Pe}} \gg 1$}

To solve (5.11) for large $v$ we apply a WKB expansion, by setting $F=\mathrm{e}^{S_{0}+S_{1}+\cdots}$ with $\left|S_{0}\right| \gg\left|S_{1}\right|$. Hence

$S_{0 Y}^{2}+v Y\left(1-\frac{1}{2} Y\right)=0$

$S_{0 Y Y}+2 S_{0 Y} S_{1 Y}=0$.

Rearranging (C.1a) gives $S_{0 Y}= \pm \mathrm{i} v^{\frac{1}{2}} \sqrt{w}$, where $w=Y(1-Y / 2)$; we define $V=\int_{0}^{Y} \sqrt{w} \mathrm{~d} Y$ to give

$S_{0}= \pm \mathrm{i} v^{\frac{1}{2}} V+B_{0}$

for some constant $B_{0}$. We rearrange (C.1b) to give

$S_{1}=\log \left(v^{-\frac{1}{4}} w^{-\frac{1}{4}}\right)+B_{1}$,

for some constant $B_{1}$. Thus we may write

$F=A(\nu w)^{-\frac{1}{4}} \exp \left[\mathrm{i} v^{\frac{1}{2}} V\right]+\bar{A}(v w)^{-\frac{1}{4}} \exp \left[-\mathrm{i} v^{\frac{1}{2}} V\right]$

where $A \in \mathbb{C}$ and $\bar{A}$ is its complex conjugate. We apply $F_{Y}=0$ at $Y=1$ to give $\bar{A}=\exp \left[2 \mathrm{i} v^{\frac{1}{2}} V(1)\right] A$, so

$F=\hat{A}(\nu w)^{-\frac{1}{4}} \cos \left[v^{\frac{1}{2}}(V(Y)-V(1))\right]$,

for some $\hat{A} \in \mathbb{R}$. This applies when $S_{0} \gg S_{1}$, i.e., for $v^{-\frac{1}{3}}(\log v)^{\frac{2}{3}} \leq Y \leq 1$, so we need another formulation for the boundary layer near the wall.

For $Y \ll 1, w \sim Y$ and $V \sim \frac{2}{3} Y^{\frac{3}{2}}$, which gives

$F_{Y Y}+v Y F=0, \quad F_{Y}=\mu_{\text {eff }} F$ on $Y=0$,

which we need to match to the inner limit of (C.5),

$F=\hat{A}(\nu Y)^{-\frac{1}{4}} \cos \left[v^{\frac{1}{2}}\left(\frac{2}{3} Y^{\frac{3}{2}}-V(1)\right)\right]$.

We put $\xi=-v^{\frac{1}{3}} Y$ into (C.6), to give

$F_{\xi \xi}-\xi F=0$,

$F_{\xi}=-\hat{\mu}_{\text {eff }} F$ on $\xi=0$,

$F \rightarrow \hat{A}\left(-v^{\frac{2}{3}} \xi\right)^{-\frac{1}{4}} \cos \left[v^{\frac{1}{2}}\left(\frac{2}{3} v^{-\frac{1}{2}} \mathrm{i} \xi^{\frac{3}{2}}-V(1)\right)\right]$ as $\xi \rightarrow-\infty$,

where $\hat{\mu}_{\text {eff }}=v^{-\frac{1}{3}} \mu_{\text {eff }}$. Equation (C.8a) has the solution $F=D_{0} \operatorname{Ai}(\xi)+D_{1} \operatorname{Bi}(\xi)$. To find values of the constants $D_{0}$ and $D_{1}$ we apply (C.8b) to give $D_{1}=-D_{0} Q$, where

$Q=\frac{\left[-3^{\frac{1}{3}} \Gamma\left(\frac{2}{3}\right)+\hat{\mu}_{\mathrm{eff}} \Gamma\left(\frac{1}{3}\right)\right]}{3^{\frac{5}{6}} \Gamma\left(\frac{2}{3}\right)+\hat{\mu}_{\mathrm{eff}} 3^{\frac{1}{2}} \Gamma\left(\frac{1}{3}\right)}$.

To apply the matching condition (C.8c), we use the asymptotic expansions

$$
\begin{aligned}
& \operatorname{Ai}(\xi) \sim \pi^{-\frac{1}{2}}(-\xi)^{-\frac{1}{4}} \sin \left(\frac{2}{3}(-\xi)^{\frac{3}{2}}+\frac{1}{4} \pi\right), \\
& \operatorname{Bi}(\xi) \sim \pi^{-\frac{1}{2}}(-\xi)^{-\frac{1}{4}} \cos \left(\frac{2}{3}(-\xi)^{\frac{3}{2}}+\frac{1}{4} \pi\right),
\end{aligned}
$$


for $\xi \rightarrow-\infty[48$, pp. 448], which gives

$F \rightarrow \frac{\sqrt{2} D_{0}}{4} \pi^{-\frac{1}{2}}(-\xi)^{-\frac{1}{4}}\left[\sin \left(\frac{2}{3} \mathrm{i} \xi^{\frac{3}{2}}\right)(1+Q)+\cos \left(\frac{2}{3} \mathrm{i} \xi^{\frac{3}{2}}\right)(1-Q)\right]$ as $\xi \rightarrow-\infty$.

This we compare with (C.8c), to give

$$
\begin{aligned}
& \hat{A} v^{-\frac{1}{6}} \sin \left(v^{\frac{1}{2}} V(1)\right)=\frac{\sqrt{2}}{4} D_{0} \pi^{-\frac{1}{2}}(1+Q), \\
& \hat{A} v^{-\frac{1}{6}} \cos \left(v^{\frac{1}{2}} V(1)\right)=\frac{\sqrt{2}}{4} D_{0} \pi^{-\frac{1}{2}}(1-Q),
\end{aligned}
$$

which we combine to give

$$
\tan \left(v^{\frac{1}{2}} V(1)\right)=(1+Q) /(1-Q),
$$

which is converted to a relationship between $\mu_{\mathrm{eff}}$ and $v$ of

$$
\hat{\mu}_{\text {eff }}=3^{\frac{1}{3}} \delta \frac{\left(1-\alpha \tan \left(v^{\frac{1}{2}} V(1)\right)\right)}{\left(-\alpha+\tan \left(v^{\frac{1}{2}} V(1)\right)\right)},
$$

where $\delta \equiv \Gamma\left(\frac{2}{3}\right) / \Gamma\left(\frac{1}{3}\right)$ and $\alpha \equiv(\sqrt{3}+1) /(\sqrt{3}-1)$. This result gives multiple eigenvalues for each $\mu_{\mathrm{eff}}$ in close agreement with numerics even for moderate values of $v$ (see Fig. 18). As $\mu_{\text {eff }} \rightarrow \infty, v_{1}$ approaches a finite-value $v_{\infty}$ which we find from (C.15) as

$v_{\infty}=\left(\tan ^{-1} \alpha / V(1)\right)^{2} \approx 5.555$..

\section{References}

1. Kunert C, Harting J (2007) Roughness induced boundary slip in microchannel flows. Phys Rev Lett 99:176001

2. Neto C, Evans DR, Bonaccurso E, Butt HJ, Craig VSJ (2005) Boundary slip in Newtonian liquids: a review of experimental studies. Rep Prog Phys 68:2859-2897

3. Taylor GI (1971) A model for the boundary condition of a porous material. Part 1. J Fluid Mech 49:319-326

4. Richardson S (1971) A model for the boundary condition of a porous material. Part 2. J Fluid Mech 49:327-336

5. Richardson S (1973) On the no-slip boundary condition. J Fluid Mech 59:707-719

6. Hocking LM (1976) A moving fluid interface on a rough surface. J Fluid Mech 76:801-817

7. Tuck EO, Kouzoubov A (1995) A laminar roughness boundary condition. J Fluid Mech 300:59-70

8. Zhou H, Khayat RE, Martinuzzi RJ, Straatman AG (2002) On the validity of the perturbation approach for the flow inside weakly modulated channels. Int J Numer Methods Fluids 39:1139-1159

9. Higdon JJL (1985) Stokes flow in arbitrary two-dimensional domains: shear flow over ridges and cavities. J Fluid Mech 159: 195-226

10. Shen C, Floryan JM (1985) Low Reynolds number flow over cavities. Phys Fluids 28:3191-3202

11. Lecoq N, Anthore R, Cichocki B, Szymczak P, Feuillebois F (2002) Drag force on a sphere moving towards a corrugated wall. J Fluid Mech 513:247-264

12. Miksis MJ, Davis SH (1994) Slip over rough and coated surfaces. J Fluid Mech 273:125-139

13. Sarkar K, Prosperetti A (1996) Effective boundary conditions for Stokes flow over a rough surface. J Fluid Mech 316:223-240

14. Tartakovsky DM, Xiu D (2006) Stochastic analysis of transport in tubes with rough walls. J Comput Phys 217:248-259

15. Stroock AD, Dertinger SK, Whitesides GM, Ajdari A (2002) Patterning flows using grooved surfaces. Anal Chem 74:5306-5312

16. Wang CY (2003) Flow over a surface with parallel grooves. Phys Fluids 15:1114-1121

17. Moffatt HK (1964) Viscous and resistive eddies near a sharp corner. J Fluid Mech 18:1-18

18. Wierschem A, Scholle M, Aksel N (2003) Vortices in film flow over strongly undulated bottom profiles at low Reynolds numbers. Phys Fluids 15:426-435

19. Scholle MI, Wierschem AI, Aksel NI (2004) Creeping films with vortices over strongly undulated bottoms. Acta Mech 168: 167-193

20. Pozrikidis C (1987) Creeping flow in two-dimensional channels. J Fluid Mech 180:495-514

21. Luchini P, Manzo F, Pozzi A (1991) Resistance of a grooved surface to parallel flow and cross-flow. J Fluid Mech 228:87-109 
22. Park JS, Hyun JM (2002) The Stokes-flow friction on a wedge surface by the sliding of a plate. Fluid Dyn Res 30:93-106

23. Phan-Thien N (1982) Hydrodynamic lubrication of rough surfaces. Proc R Soc Lond A Mat 383:439-446

24. Achdou Y, Pironneau O, Valentin F (1998) Effective boundary conditions for laminar flows over periodic rough boundaries. J Comput Phys 147:187-218

25. Brady M, Pozrikidis C (1993) Diffusive transport across irregular and fractal walls. Proc R Soc Lond A Math 442:571-583

26. Blyth MG, Pozrikidis C (2003) Heat conduction across irregular and fractal-like surfaces. Int J Heat Mass Transf 46:1329-1339

27. Fyrillas MM, Pozrikidis C (2001) Conductive heat transport across rough surfaces and interfaces between two conforming media. Int J Heat Mass Transf 44:1789-1801

28. Neagu M, Bejan A (2001) Constructal placement of high-conductivity inserts in a slab: optimal design of roughness. J Heat Transf 123:1184

29. Horner M, Metcalfe G, Wiggins S, Ottino JM (2002) Transport enhancement mechanisms in open cavities. J Fluid Mech 452: 199-229

30. Laine-Pearson FE, Hydon PE (2006) Particle transport in a moving corner. J Fluid Mech 559:379-390

31. Weber JEH (2005) Mean drift velocity in viscous flow over a corrugated bottom. Phys Fluids 17:113102

32. Smith JW, Gowen RA (1965) Heat transfer efficiency in rough pipes at high Prandtl number.. A I Ch E J 11:941-943

33. Sobey IJ (1980) On flow through furrowed channels. Part 1. J Fluid Mech 96:1-26

34. Russ G, Beer H (1997) Heat transfer and flow field in a pipe with sinusoidal wavy surface- I. Numerical investigation. Int J Heat Mass Transf 40:1061-1070

35. Wang CC, Chen CK (2002) Forced convection in a wavy-wall channel. Int J Heat Mass Transf 45:2587-2595

36. Das PK, Mahmud S, Tasnim SH, Islam A (2003) Effect of surface waviness and aspect ratio on heat transfer inside a wavy enclosure. Int J Numer Method H 13:1097-1122

37. Nevard J, Keller JB (1997) Homogenization of rough boundaries and interfaces. SIAM J Appl Math 57:1660-1686

38. Szumbarski J, Floryan JM (1999) A direct spectral method for determination of flows over corrugated boundaries. J Comput Phys 153:378-402

39. Amirat Y, Bodart O, De Maio U, Gaudiello A (2004) Asymptotic approximation of the solution of the Laplace equation in a domain with highly oscillating boundary. SIAM J Math Anal 35:1598-1616

40. Amidon GL, Kou J, Elliott RL, Lightfoot EN (1980) Analysis of models for determining intestinal wall permeabilities. J Pharm Sci 69:1369-1373

41. Wilding I (2000) Site-specific drug delivery in the gastrointestinal tract. Crit Rev Ther Drug Carrier Syst 17:557-620

42. Wacher VJ, Salphati L, Benet LZ (2001) Active secretion and enterocytic drug metabolism barriers to drug absorption. Adv Drug Dely Rev 46:89-102

43. Stephens RH, Tanianis-Hughes J, Higgs NB, Humphrey M, Warhurst G (2002) Region-dependent modulation of intestinal permeability by drug efflux transporters: In vitro ttudies in mdr1a (-/-) mouse intestine. J Pharm Exp Ther 303:1095-1101

44. Žakelj S, Šturm K, Kristl A (2006) Ciprofloxacin permeability and its active secretion through rat small intestine in vitro. Int J Phar 313:175-180

45. Burton PS, Goodwin JT, Vidmar TJ, Amore BM (2002) Predicting drug absorption: how nature made it a difficult problem. J Pharm Exp Ther 303:889-895

46. Stoll BR, Batycky RP, Leipold HR, Milstein S, Edwards DA (2000) A theory of molecular absorption from the small intestine. Chem Eng Sci 55:473-489

47. Grotberg JB, Jensen OE (2004) Biofluid mechanics in flexible tubes. Annu Rev Fluid Mech 36:121-147

48. Abramowitz M, Stegun IA (eds) (1965) Handbook of Mathematical Functions. Dover Publications, New York 